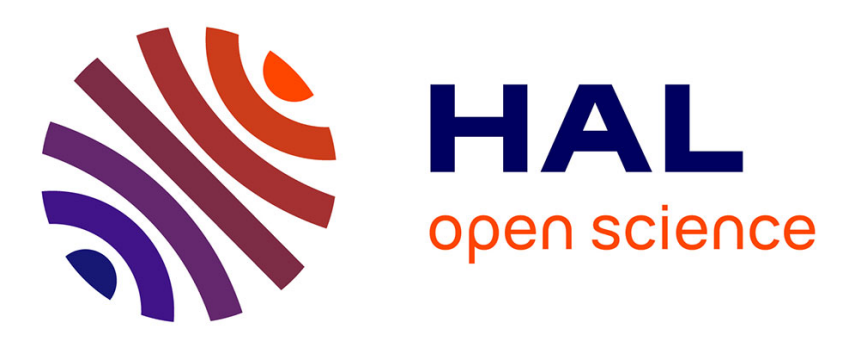

\title{
The Variational Theory of Complex Rays for three-dimensional Helmholtz problems
}

Louis Kovalevsky, Pierre Ladevèze, Hervé Riou, Marc Bonnet

\section{To cite this version:}

Louis Kovalevsky, Pierre Ladevèze, Hervé Riou, Marc Bonnet. The Variational Theory of Complex Rays for three-dimensional Helmholtz problems. Journal of Computational Acoustics, 2012, 20, 125021 (25 p.). 10.1142/S0218396X1250021X . hal-00714312

\section{HAL Id: hal-00714312 https://hal.science/hal-00714312}

Submitted on 4 Jul 2012

HAL is a multi-disciplinary open access archive for the deposit and dissemination of scientific research documents, whether they are published or not. The documents may come from teaching and research institutions in France or abroad, or from public or private research centers.
L'archive ouverte pluridisciplinaire HAL, est destinée au dépôt et à la diffusion de documents scientifiques de niveau recherche, publiés ou non, émanant des établissements d'enseignement et de recherche français ou étrangers, des laboratoires publics ou privés. 


\title{
The Variational Theory of Complex Rays for three-dimensional Helmholtz problems
}

\author{
Louis KOVALEVSKY \\ LMT Cachan,Ens Cachan/UPMC/CNRS/PRES UniverSud Paris \\ 61 avenue du Président Wilson - 94230 Cachan, France \\ kovalevsky@lmt.ens-cachan.fr \\ Pierre LADEVÈZE \\ EADS Foundation Chair Advanced Computational Structural Mechanics \\ LMT Cachan,Ens Cachan/UPMC/CNRS/PRES UniverSud Paris \\ 61 avenue du Président Wilson - 94230 Cachan, France \\ ladeveze@lmt.ens-cachan.fr \\ Hervé RIOU \\ LMT Cachan,Ens Cachan/UPMC/CNRS/PRES UniverSud Paris \\ 61 avenue du Président Wilson - 94230 Cachan, France \\ riou@lmt.ens-cachan.fr \\ Marc BONNET \\ POems (UMR 7231 CNRS-ENSTA-INRIA) \\ 32 boulevard Victor, 75015 Paris, France \\ marc.bonnet@ensta.fr \\ Received (Day Month Year) \\ Revised (Day Month Year)
}

This article proposes an extension of the Variational Theory of Complex Rays (VTCR) to threedimensional linear acoustics, The VTCR is a Trefftz-type approach designed for mid-frequency range problems and has been previously investigated for structural dynamics and 2D acoustics. The proposed 3D formulation is based on a discretization of the amplitude portrait using spherical harmonics expansions. This choice of discretization allows to substantially reduce the numerical integration work by taking advantage of well-known analytical properties of the spherical harmonics. It also permits (like with the previous 2D Fourier version) an effective a priori selection method for the discretization parameter in each sub-region, and allows to estimate the directivity of the pressure field by means of a natural definition of rescaled amplitude portraits. The accuracy and performance of the proposed formulation are demonstrated on a set of numerical examples that include results on an actual case study from the automotive industry.

Keywords: Mid-frequency; Helmholtz equation; Acoustics; Variational Theory of Complex Rays; Fourier series; Spherical harmonics 


\section{Introduction}

Numerical simulation techniques in design, analysis and optimization of systems are nowadays an essential part of the industrial design process. The standard Galerkin finite element method $\left(\mathrm{FEM}^{1}\right)$, a well established computer aided engineering tool, is in particular commonly used for the analysis of time-harmonic dynamic problems. However, the use of continuous, piecewise-polynomial shape functions leads to very large numerical models and, in practice, restricts the application of the standard FEM to the low-frequency range. Moreover, since the shape functions are not exact solutions of the governing partial differential equations, fine discretization is required to alleviate the associated pollution error ${ }^{2}$.

Over the recent years, a variety of techniques have been proposed to minimize these drawbacks and increase the practical range of application of the FEM to higher frequencies. Such techniques include the predefined reduced bases ${ }^{3}$, the Galerkin least-squares $\mathrm{FEM}^{4}$, the quasi-stabilized finite element method ${ }^{5}$, the partition of unity $\operatorname{method}^{6}$, the generalized finite element method ${ }^{7}$, residual-free bubbles ${ }^{8}$, and the quasi optimal Petrov-Galerkin $\operatorname{method}^{9}$. While all these methods have established abilities for reducing computational costs, their permitted frequency ranges remain below the mid- and high frequencies.

In addition to all the previously mentioned approaches, another family of solution algorithms is based on the Trefftz methods ${ }^{10}$, which differ from FEM-type methods by expanding the unknown fields as combinations of basis functions that exactly satisfy the relevant governing partial differential equations (PDEs). Such approaches also include, for example, a special use of the partition of unity $\operatorname{method}^{11}$, the ultra-weak variational method $^{12}$, the least-squares method ${ }^{13}$, the discontinuous enrichment method ${ }^{14}$, the element-free Galerkin $\operatorname{method}^{15}$, the wave boundary element $\operatorname{method}^{16}$ and the wave-based method ${ }^{17}$. The Variational Theory of Complex Rays (VTCR), which is addressed in this paper, also belongs to this family.

The main advantage of all Trefftz methods is that no refined discretization is necessary, as they use exact solutions of the governing PDE. As a result, the model size and computational effort are considerably reduced compared to element-based methods. The main differences among the various Trefftz methods lie in the treatment of the boundary conditions and the transmission conditions between sub-regions, and in the type of shape functions used.

The VTCR is based on a specially-developed weak formulation of the problem which allows the approximations in each sub-region to be a priori independent of one another $\left(\right.$ see $\left.^{18}\right)$. Thus, in each sub-region, any type of shape function can be used provided it verifies the governing PDE. This lends great flexibility, and thus efficiency, to the approach as basis functions having clear mechanical meaning can be used without difficulty. For the acoustic vibration problems considered in this article, the VTCR approximates the solution through an integral superposition of plane waves, also known as a Herglotz wave function, ensuring exact satisfaction of the Helmholtz equation within each sub-domain. However, these functions usually violate boundary or transmission conditions. The VTCR is designed so as to enforce all boundary or transmission conditions in a weak sense, i.e. set to zero the projections of the boundary or transmission condition residuals onto the Herglotz wave 
functions. This process yields a matrix equation of small size, whose solution gives the angular distribution of plane waves in each sub-region. This approach has initially been proposed for mid-frequency vibrational analyses of structures, e.g. to predict the vibrational response of a 3 -D plate assembly ${ }^{19,20}$, and has later been extended to shells ${ }^{21}$ and heterogeneous plates $^{22}$, the computation of vibrational responses ${ }^{23}$, and transient structural dynamics ${ }^{24}$.

More recently, the VTCR has also been considered for computing acoustic fields ${ }^{25}$, and an adaptive version subsequently formulated ${ }^{26}$. Its ability to provide accurate solutions within moderate problem sizes was demonstrated through many examples. A discretization of the Herglotz wave function based on Fourier series was later proposed ${ }^{27}$, allowing in particular to develop efficient semi-analytical techniques for the discrete VTCR problem setup and an effective a priori discretization criterion. An error estimator based on dissipated energy has also been investigated ${ }^{26,27}$.

Thus far the implementation of the linear-acoustic VTCR has been confined to 2D situations. The main goal of the present article is to propose for the first time a computationallyefficient 3D version of the linear-acoustic VTCR. The main practical difficulty faced a priori by $3 \mathrm{D}$ implementations is the potentially high numerical quadrature costs entailed by the computation of six-dimensional integrals entering the VTCR matrix entries if a fullynumerical approach is followed. This issue arises because typical integrals contributions consist of surface integrals featuring products of shape functions, each of which being defined as an integral over the unit sphere. To resolve this issue, it is proposed here to express the densities entering the Herglotz wave functions, i.e. the shape functions upon which the approximation space is built, using spherical harmonics. This is in fact the natural extension to 3D problems of the previously-developed Fourier approach for 2D acoustics. Exploitation of the analytical properties of the spherical harmonics, and in particular of their $L^{2}$-orthonormality on the unit sphere, permits analytical evaluation of all shape functions and reduce numerical quadratures from six to two dimensions. This treatment is shown here to yield an accurate and computationally efficient method, and also to permit (like with the previous 2D Fourier version) an effective a priori selection method for the discretization parameter in each sub-region.

This article is organized as follows. The VTCR formulation is first reviewed in Section 2. Then, the proposed 3-D version of VTCR based on a spherical harmonics decomposition of the Herglotz wave function density is presented in Section 3. A set of numerical examples designed to study the accuracy and performance of the proposed formulation, as well as results from an actual case study, are presented in Section 4. Finally, concluding remarks are given in Section 5 .

\section{The Variational Theory of Complex Rays for Helmholtz problems}

\subsection{The reference problem}

Consider the general three-dimensional interior problem for an acoustic cavity $\Omega$ filled with a fluid characterized by its speed of sound $c$, its density $\rho$ and its damping coefficient $\eta$. Assuming all excitations to be time-harmonic with a given circular frequency $\omega$, the acoustic 
pressure $p$ in $\Omega$ solves the following boundary-value problem for the Helmholtz equation:

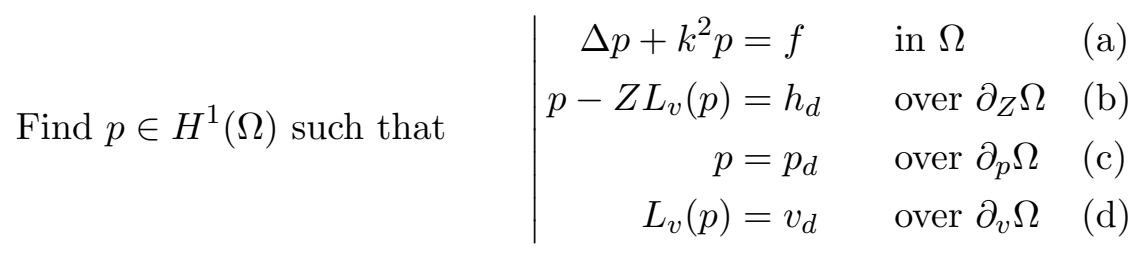

where $k=(1+\mathrm{i} \eta)(\omega / c)=(1+\mathrm{i} \eta) k_{0}$ is the wave number, $f$ a given source density, $Z$ an impedance coefficient and $L_{v}(\square)$ the operator expressing the normal velocity in terms of the pressure, defined by $L_{v}(\square)=\mathrm{i}\left(\rho_{0} \omega\right)^{-1} \mathbf{n} \cdot \nabla(\square)$, $\mathbf{n}$ being the outward normal to $\partial \Omega$. $h_{d}$, $p_{d}$ and $v_{d}$ denote respectively a prescribed excitation over $\partial_{Z} \Omega$, a prescribed pressure over $\partial_{p} \Omega$ and a prescribed normal velocity over $\partial_{v} \Omega$. The usual complex-valued representation of physical quantities is used throughout, whereby the complex amplitude $Q(\mathbf{x})$ is associated to any physical quantity of the form $\operatorname{Re}\left[Q(\mathbf{x}) e^{-\mathrm{i} \omega t}\right]$ evaluated at spatial position $\mathbf{x}$ and time $t$ (with $\mathrm{i}=\sqrt{-1}$ denoting the imaginary unit).

Let $\Omega$ be partitioned into $N_{\Omega}$ non-overlapping sub-cavities $\Omega_{E}\left(1 \leq E \leq N_{\Omega}\right)$, and set $\Gamma_{E, E^{\prime}}=\partial \Omega_{E} \cap \partial \Omega_{E^{\prime}}$. The reference problem (1) is then rewritten in terms of the acoustic pressure $p_{E}$ in each sub-cavity $\Omega_{E}$ :

Find $\left(p_{1}, \ldots, p_{N_{\Omega}}\right) \in H^{1}\left(\Omega_{1}\right) \times \cdots \times H^{1}\left(\Omega_{N_{\Omega}}\right)$ such that:

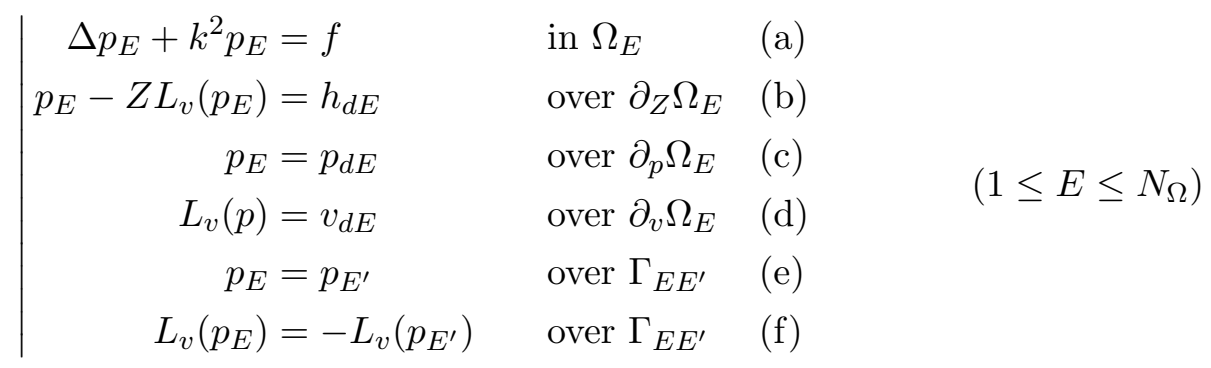

where conditions $(2 \mathrm{e}, \mathrm{f})$ express the continuity conditions across $\Gamma_{E, E^{\prime}}$ and $p_{E}, Z_{E}, p_{d E}$, $h_{d E}$ and $v_{d E}$ respectively denote the pressure, the impedance coefficient and the prescribed excitations for the cavity $\Omega_{E}$ (i.e. the restrictions of $p, Z, p_{d}, h_{d}$ and $v_{E}$ to $\Omega_{E}$ ).

\subsection{The VTCR formulation}

The VTCR formulation is obtained from the boundary value problem (2) by rewriting it in a weak form. For this purpose, the following spaces $S_{a d, 0}^{E}$ and $S_{a d}^{E}$ of functions satisfying the homogeneous and non-homogeneous Helmholtz equation (i.e. equation (1a) with $f=0$ or $f \neq 0)$, respectively, in each sub-cavity $\Omega_{E}$ are introduced:

$$
S_{a d, 0}^{E}=\left\{p_{E} \in H^{1}\left(\Omega_{E}\right) \quad \mid \quad \Delta p_{E}+k^{2} p_{E}=0 \text { in } \Omega_{E}\right\}, \quad S_{a d}^{E}=S_{a d, 0}^{E}+p_{E}^{p}
$$

where $p_{E}^{p}$ is a particular solution of $\Delta p_{E}+k^{2} p_{E}=f\left(\right.$ e.g. $p_{E}^{p}(\mathbf{x})=\int_{\Omega_{E}} f(\mathbf{x}) G(\mathbf{x}, \mathbf{y}) \mathrm{d} V(\mathbf{y})$, where $G(\mathbf{x}, \mathbf{y})$ is the Green's function for the Helmholtz equation). 
The VTCR formulation is:

Find $\left(p_{1}, p_{2}, \cdots, p_{N_{\Omega}}\right) \in S_{a d}^{1} \times S_{a d}^{E} \times \cdots \times S_{a d}^{N_{\Omega}}$ such that:

$$
\begin{gathered}
\operatorname{Re}\left\{\frac{1}{2} \sum_{E=1}^{N_{\Omega}} \int_{\partial_{Z} \Omega_{E}}\left(p_{E}-Z L_{v}\left(p_{E}\right)-h_{d E}\right) \overline{L_{v}\left(\delta p_{E}\right)}+\frac{1}{\bar{Z}} \overline{\left(p_{E}-Z L_{v}\left(p_{E}\right)-h_{d E}\right)} \delta p_{E} \mathrm{~d} S\right. \\
+\sum_{E=1}^{N_{\Omega}} \int_{\partial_{p} \Omega_{E}}\left(p_{E}-p_{d E}\right) \overline{L_{v}\left(\delta p_{E}\right)} \mathrm{d} S+\sum_{E=1}^{N_{\Omega}} \int_{\cap_{\partial_{v} \Omega_{E}}} \overline{L_{v}\left(p_{E}\right)-v_{d E}} \delta p_{E} \mathrm{~d} S \\
\left.+\sum_{E, E^{\prime}<E} \frac{1}{2} \int_{\Gamma_{E, E^{\prime}}}\left(\left(p_{E}-p_{E^{\prime}}\right) \overline{L_{v}\left(\delta p_{E}-\delta p_{E^{\prime}}\right)}+\overline{L_{v}\left(p_{E}+p_{E^{\prime}}\right)} \cdot\left(\delta p_{E}+\delta p_{E^{\prime}}\right)\right) \mathrm{d} S\right\}=0 \\
\forall\left(\delta p_{1}, \delta p_{2}, \cdots, \delta p_{N_{\Omega}}\right) \in S_{a d, 0}^{1} \times S_{a d, 0}^{2} \times \cdots \times S_{a d, 0}^{N_{\Omega}}
\end{gathered}
$$

This formulation has been shown earlier ${ }^{27}$ to be equivalent to the reference problem (1ad) if $\eta>0$ or $\operatorname{Re}(Z)>0$ (either condition corresponding to a form of damping). In the absence of dissipation, this equivalence still holds unless at frequencies that permit nonzero pressure fields satisfying the continuity conditions $(2 \mathrm{e}, \mathrm{f})$ and homogeneous versions of the boundary conditions ( $1 \mathrm{~b}-\mathrm{d})$, i.e. eigenfrequencies for the whole domain $\Omega$ subject to the latter boundary conditions.

The VTCR formulation (4) essentially consists in enforcing in a weak sense the boundary and transmission condition between subcavities. It leads to a non-symmetric linear problem. This makes the method very flexible as approximation spaces can be constructed independently for each sub-cavity, allowing the user to choose physically meaningful basis functions.

Symmetric formulations for Trefftz problems are nevertheless also available ${ }^{14}$. They involve Lagrange multipliers, whose precise definition depends on the chosen approximation space for the pressure, resulting in larger problem sizes and increased implementation technicality. The VTCR thus emphasizes flexibility of approximation and ease of implementation over symmetry.

In practice, one sets $p_{E}=p_{E}^{0}+p_{E}^{p}$ in (4) and recasts it in terms of the unknowns $p_{E}^{0}$, with the known particular solution $p_{E}^{p}$ contributing to the right-hand side. In the sequel, this modification will be implied throughout and attention will be focused on solving for $p_{E}^{0}\left(1 \leq E \leq N_{\Omega}\right)$ the VTCR formulation.

\subsection{Finite-dimensional approximation space}

To find approximate solutions to VCTR formulations (4), $p_{E}$ is sought in each cavity $\Omega_{E}$ in the form $p_{E}=p_{E}^{0}+p_{E}^{p}$, where $p_{E}^{p}$ is, as discussed above, a known particular solution while $p_{E}^{0} \in S_{a d, 0}^{E}$ is taken as a Herglotz wave function, i.e. an integral superposition of plane waves of the form

$$
\mathcal{H}\left[A_{E}\right](\mathbf{x}):=\int_{\mathcal{C}} A_{E}(\hat{\mathbf{s}}) e^{\mathrm{i} k \hat{\mathbf{s}} \cdot\left(\mathbf{x}-\mathbf{x}_{E}\right)} \mathrm{d} \hat{\mathbf{s}} .
$$

In (5), $\mathcal{C}$ is the unit sphere (or, for previous two-dimensional implementations of the VTCR, the unit circle), $\mathbf{x}_{E}$ is a reference point located in $\Omega_{E}$ and $A_{E}(\hat{\mathbf{s}})$ is the amplitude of the plane 
wave propagating in the direction $\hat{\mathbf{s}}$ that contributes to $p_{E}$. Any Herglotz wave function is a solution to the homogeneous Helmholtz equation. Moreover, it is shown elsewhere ${ }^{28}$ that, for cavities $\Omega_{E}$ with smooth $C^{2}$ boundary, the space of all Herglotz wave functions with density $A_{E} \in L^{2}(\mathcal{C})$ (i.e. for which $A_{E}$ is square-integrable over $\mathcal{C}$ ) is dense with respect to the $H^{1}\left(\Omega_{E}\right)$ norm in the space of all solutions to the Helmholtz equation. This mathematical result, which holds for any frequency ${ }^{28}$, thus guarantees that $p_{E}$ may be approximated within arbitrary accuracy by a Herglotz wave function. Keeping terminology adopted in previous work on the VCTR, the density $A_{E}$ is called the amplitude portrait of $p_{E}$.

To achieve a discrete version of the VCTR formulation (4), finite-dimensional subspaces $S_{a d, 0}^{E, N_{E}}$ of $S_{a d, 0}^{E}$ (with $N_{E}$ denoting a discretization parameter to be specified) are then constructed by discretizing the amplitude portraits $A_{E}$ entering Herglotz wave functions $\mathcal{H}\left[A_{E}\right]$. For instance, the following approaches have been considered in previous two-dimensional implementations of the $\mathrm{VTCR}^{25,27}$, for which $\mathcal{C}$ is the unit circle:

- $A_{E}(\hat{\mathbf{s}})$ is a sum of $N_{E}$ Dirac distributions supported at angular locations $\theta_{n}, p_{E}$ then being the superposition of a finite number of plane waves:

$$
S_{a d, 0}^{E, N_{E}}=\operatorname{Span}\left\{e^{\mathrm{i} k\left(\left(x-x_{E}\right) \cdot \cos \theta_{n}+\left(y-y_{E}\right) \cdot \sin \theta_{n}\right)}, \quad n=0, \ldots, N_{E}-1, \quad \theta_{n}=\frac{2 \pi n}{N_{E}}\right\} .
$$

In this case, $\operatorname{Dim}\left(S_{a d, 0}^{E, N_{E}}\right)=N_{E}$. This discretization presents the main advantage to allow analytical integration of the components of the weak formulation (4) on straight lines, but loses the continuous-superposition feature.

- $A_{E}(\hat{\mathbf{s}})$ is taken as piecewise-constant on $\mathcal{C}$ function, $p_{E}$ then being a superposition of wave band functions:

$$
S_{a d, 0}^{E, N_{E}}=\operatorname{Span}\left\{\int_{\theta_{n}}^{\theta_{n+1}} e^{\mathrm{i} k\left[\left(x-x_{E}\right) \cdot \cos \theta+\left(y-y_{E}\right) \cdot \sin \theta\right]} \mathrm{d} \theta, \quad n=0, \ldots, N_{E}-1, \quad \theta_{n}=\frac{2 \pi n}{N_{E}}\right\} .
$$

One also has $\operatorname{Dim}\left(S_{a d, 0}^{E, N_{E}}\right)=N_{E}$. This discretization retains the continuoussuperposition feature, but analytical integration in (4) is not feasible even with straight lines.

- $A_{E}(\hat{\mathbf{s}})$ is taken as a truncated Fourier series, $p_{E}$ then being a superposition of Fourier wave functions:

$$
S_{a d, 0}^{E, N_{E}}=\operatorname{Span}\left\{\int_{-\pi}^{\pi} e^{\mathrm{i} n \theta} e^{i k\left(\left(x-x_{E}\right) \cdot \cos \theta+\left(y-y_{E}\right) \cdot \sin \theta\right)} \mathrm{d} \theta, \quad n=-N_{E}, \ldots, N_{E}\right\} .
$$

In this case, $\operatorname{Dim}\left(S_{a d, 0}^{E, N_{E}}\right)=2 N_{E}+1$. The main advantages of this discretization is that the continuous-superposition feature is preserved, while the shape functions $\mathcal{H}\left[A_{E}\right](\mathbf{x})$ can be evaluated analytically at any point $\mathbf{x}$. Moreover these functions make a hierarchical basis of functions, as they are nested.

The above discretizations have their pros and cons, and lead to very similar asymptotic accuracy, as seen in comparisons presented elsewhere ${ }^{25,27}$. For cases (6) and (7), the discretization parameter $N_{E}$, and hence $\operatorname{Dim}\left(S_{a d, 0}^{E, N_{E}}\right)$, can be set a priori using a geometrical heuristic criterion: the number of shape functions must be proportional to the ratio $k R_{E} /(2 \pi)$ of the 
characteristic diameter $R_{E}$ of $\Omega_{E}$ and the wavelength (see ${ }^{29}$, eqn. 43 or $^{30}$, eqn. 3.37). For case $(8), N_{E}$ can be set a priori using an energetic criterion $\left(\mathrm{see}^{27}\right)$.

\section{The 3-D version of VTCR with spherical harmonics decomposition of plane waves amplitudes}

The three-dimensional version of the VTCR for Helmholtz problems, which is the main focus of this article, is now developed. The discrete formulation is based on expanding the amplitude portraits over the spherical harmonics. In addition to being the natural extension to $3 \mathrm{D}$ problems of the previously-developed Fourier approach for $2 \mathrm{D}$ acoustics, this choice will be seen to permit an accurate and efficient implementation.

\subsection{Pressure field approximation}

As indicated in section 2.3, the pressure field $p_{E}^{0}$ in each cavity is sought as a Herglotz wave function. Introducing a parametrization of the unit sphere $\mathcal{C}$ in terms of angular spherical coordinates $(\theta, \varphi) \in[0, \pi] \times[-\pi, \pi]$, i.e. $\hat{\mathbf{s}}=\hat{\mathbf{s}}(\theta, \varphi)$ into (5), one sets:

$$
p_{E}^{0}(\mathbf{x})=\mathcal{H}\left[A_{E}\right](\mathbf{x}):=\int_{0}^{\pi}\left\{\int_{-\pi}^{\pi} A_{E}(\theta, \varphi) e^{\mathrm{i} k \hat{\mathbf{s}}(\theta, \varphi) \cdot\left(\mathbf{x}-\mathbf{x}_{E}\right)} \mathrm{d} \varphi\right\} \sin \theta \mathrm{d} \theta, \quad \mathbf{x} \in \Omega_{E}
$$

where $A_{E}(\theta, \varphi)$ is the amplitude of the plane-wave contribution to $p_{E}^{0}$ propagating in the $\hat{\mathbf{s}}(\theta, \varphi)$ direction.

In order to avoid a numerical quadrature over $\mathcal{C}$ (required if using e.g. ray or wave-band shape functions) and extend to the $3 \mathrm{D}$ case the advantages of the Fourier decomposition previously introduced ${ }^{27}$ for $2 \mathrm{D}$ problems, amplitude portraits $A_{E}(\theta, \varphi)$ are represented as truncated Laplace series, which can be considered as the natural extension to 3D problems

of the Fourier series (8). Accordingly, the discrete space $S_{a d, 0}^{E, N_{E}}$ is now taken as:

$$
\begin{aligned}
S_{a d, 0}^{E, N_{E}} & =\operatorname{Span}\left\{\Phi_{\ell}^{m}(\mathbf{x}), \quad-\ell \leq m \leq \ell, 0 \leq \ell \leq N_{E}\right\} \\
\text { with } \quad \Phi_{\ell}^{m}(\mathbf{x}) & :=\int_{0}^{\pi}\left\{\int_{-\pi}^{\pi} Y_{\ell}^{m}(\theta, \varphi) e^{\mathrm{i} k \hat{\mathbf{s}}(\theta, \varphi) \cdot\left(\mathbf{x}-\mathbf{x}_{E}\right)} \mathrm{d} \varphi\right\} \sin \theta \mathrm{d} \theta
\end{aligned}
$$

where $Y_{\ell}^{m}(\theta, \varphi)=Y_{\ell}^{m}(\hat{\mathbf{s}})$ denotes the spherical harmonic of (non-negative) order $\ell$ and index $m$, given by

$$
\begin{gathered}
Y_{\ell}^{m}(\theta, \varphi)=\sqrt{\frac{2 \ell+1}{4 \pi} \frac{(l-m) !}{(l+m) !}} P_{\ell}^{m}(\cos \theta) e^{\mathrm{i} m \varphi} \\
\text { with } \quad P_{\ell}^{m}(u)=(-1)^{m}\left(1-u^{2}\right)^{m / 2} \frac{d^{m}}{d u^{m}} P_{\ell}(u) \quad(-\ell \leq m \leq \ell)
\end{gathered}
$$

where $P_{\ell}$ are the Legendre polynomials and $P_{\ell}^{m}$ are associated Legendre functions (see e.g. Secs. 8.81 and 8.91 of $^{31}$ ). Amplitude portraits are thus sought in the form:

$$
A_{E}(\theta, \varphi)=\sum_{\ell=0}^{N_{E}} \sum_{m=-\ell}^{\ell} a_{\ell m}^{E} Y_{\ell}^{m}(\theta, \varphi)
$$


The set $\left\{Y_{\ell}^{m}(\theta, \varphi)\right\}_{|m| \leqslant \ell \leqslant \infty}$ forms a complete orthogonal system on $\mathcal{C}$ for the $L^{2}(\mathcal{C})$ scalar product, denoted $(\cdot, \cdot)_{L^{2}(\mathcal{C})}$, and verifies

$$
\begin{aligned}
\left(Y_{\ell}^{m}, \bar{Y}_{\ell^{\prime}}^{m^{\prime}}\right)_{L^{2}(\mathcal{C})}=\int_{0}^{\pi}\left\{\int_{-\pi}^{\pi} Y_{\ell}^{m}(\theta, \varphi) \bar{Y}_{\ell^{\prime}}^{m^{\prime}}(\theta, \varphi) \mathrm{d} \varphi\right\} \sin \theta \mathrm{d} \theta=\delta_{\ell \ell^{\prime}} \delta_{m m^{\prime}} & \left\|Y_{\ell}^{m}\right\|_{L^{2}(\mathcal{C})}^{2}=1 .
\end{aligned}
$$

Since the $Y_{\ell}^{m}$ are in particular linearly independent, the discrete space $S_{a d, 0}^{E, N_{E}}$ defined by (10) has dimension $1+3+\ldots+\left(2 N_{E}+1\right)=\left(N_{E}+1\right)^{2}$.

\subsection{Evaluation of the shape functions}

Spherical harmonics provide the natural representation of (square-integrable) amplitude portraits defined on $\mathcal{C}$. In particular, they allow analytical evaluation of the shape functions $\Phi_{\ell}^{m}(\mathbf{x})$ at any point $\mathbf{x}$ of the sub-cavity. This is achieved by invoking the Jacobi-Anger expansion $^{32}$, whereby a plane wave is written as an expansion in spherical harmonics:

$$
e^{i k \hat{\mathbf{s}} \cdot \mathbf{x}}=4 \pi \sum_{\ell=0}^{+\infty} \sum_{m=-\ell}^{\ell} \mathrm{i}^{\ell} j_{\ell}(k r) Y_{\ell}^{m}(\hat{\mathbf{s}}) \bar{Y}_{\ell}^{m}(\hat{\mathbf{x}})
$$

where $\mathbf{x}$ is a vector with a modulus $r$ and direction $\hat{\mathbf{x}}:=\mathbf{x} / r$ and $j_{\ell}$ is the spherical Bessel function of first kind and order $\ell$, linked to the Bessel function of first kind through $j_{\ell}(t)=\sqrt{\pi /(2 t)} J_{\ell+1 / 2}(t)$.

Then, denoting by $\left(r_{x}, \theta_{x}, \phi_{x}\right)$ the spherical coordinates associated to the relative position vector $\mathbf{x}-\mathbf{x}_{E}$ (i.e. setting $\mathbf{x}-\mathbf{x}_{E}=r_{x} \hat{\mathbf{x}}\left(\theta_{x}, \varphi_{x}\right)$ ) and using the orthonormality property (12), explicit expressions for the the shape functions $\Phi_{\ell}^{m}(\mathbf{x})$ defined by (10) are obtained:

$$
\begin{aligned}
\Phi_{\ell}^{m}(\mathbf{x}) & =\int_{0}^{\pi}\left\{\int_{-\pi}^{\pi} Y_{\ell}^{m}(\theta, \varphi) e^{\mathrm{i} k \hat{\mathbf{s}}(\theta, \varphi) \cdot\left(\mathbf{x}-\mathbf{x}_{E}\right)} \mathrm{d} \varphi\right\} \sin \theta \mathrm{d} \theta \\
& =\int_{0}^{\pi}\left\{\int_{-\pi}^{\pi} Y_{\ell}^{m}(\theta, \varphi)\left[4 \pi \sum_{\ell^{\prime}=0}^{+\infty} \sum_{m^{\prime}=-\ell^{\prime}}^{\ell^{\prime}} \mathrm{i}^{\ell^{\prime}} j_{\ell^{\prime}}\left(k r_{x}\right) Y_{\ell^{\prime}}^{m^{\prime}}\left(\theta_{x}, \varphi_{x}\right) \bar{Y}_{\ell^{\prime}}^{m^{\prime}}(\theta, \varphi)\right] \mathrm{d} \varphi\right\} \sin \theta \mathrm{d} \theta \\
& =4 \pi \sum_{\ell^{\prime}=0}^{+\infty} \sum_{m^{\prime}=-\ell^{\prime}}^{\ell^{\prime}} \mathrm{i}^{\ell^{\prime}} j_{\ell^{\prime}}\left(k r_{x}\right) Y_{\ell^{\prime}}^{m^{\prime}}\left(\theta_{x}, \varphi_{x}\right) \int_{0}^{\pi}\left\{\int_{-\pi}^{\pi} Y_{\ell}^{m}(\theta, \varphi) \bar{Y}_{\ell^{\prime}}^{m^{\prime}}(\theta, \varphi) \mathrm{d} \varphi\right\} \sin \theta \mathrm{d} \theta \\
& \left.=4 \pi \sum_{\ell^{\prime}=0}^{+\infty} \sum_{m^{\prime}=-\ell^{\prime}}^{\ell^{\prime}} \mathrm{i}^{\ell^{\prime}} j_{\ell^{\prime}}\left(k r_{x}\right) Y_{\ell^{\prime}}^{m^{\prime}}\left(\theta_{x}, \varphi_{x}\right) \delta_{\ell \ell^{\prime}} \delta_{m m^{\prime}} \quad \text { by }(12)\right) \\
& =4 \pi \mathrm{i}^{\ell} j_{\ell}\left(k r_{x}\right) Y_{\ell}^{m}\left(\theta_{x}, \varphi_{x}\right)
\end{aligned}
$$

The spherical Bessel functions and spherical harmonics entering the above explicit expression of the shape functions $\Phi_{\ell}^{m}(\mathbf{x})$ can then be very efficiently evaluated numerically using low-level programming libraries. For illustration purposes, the spatial variation (14) of shape 


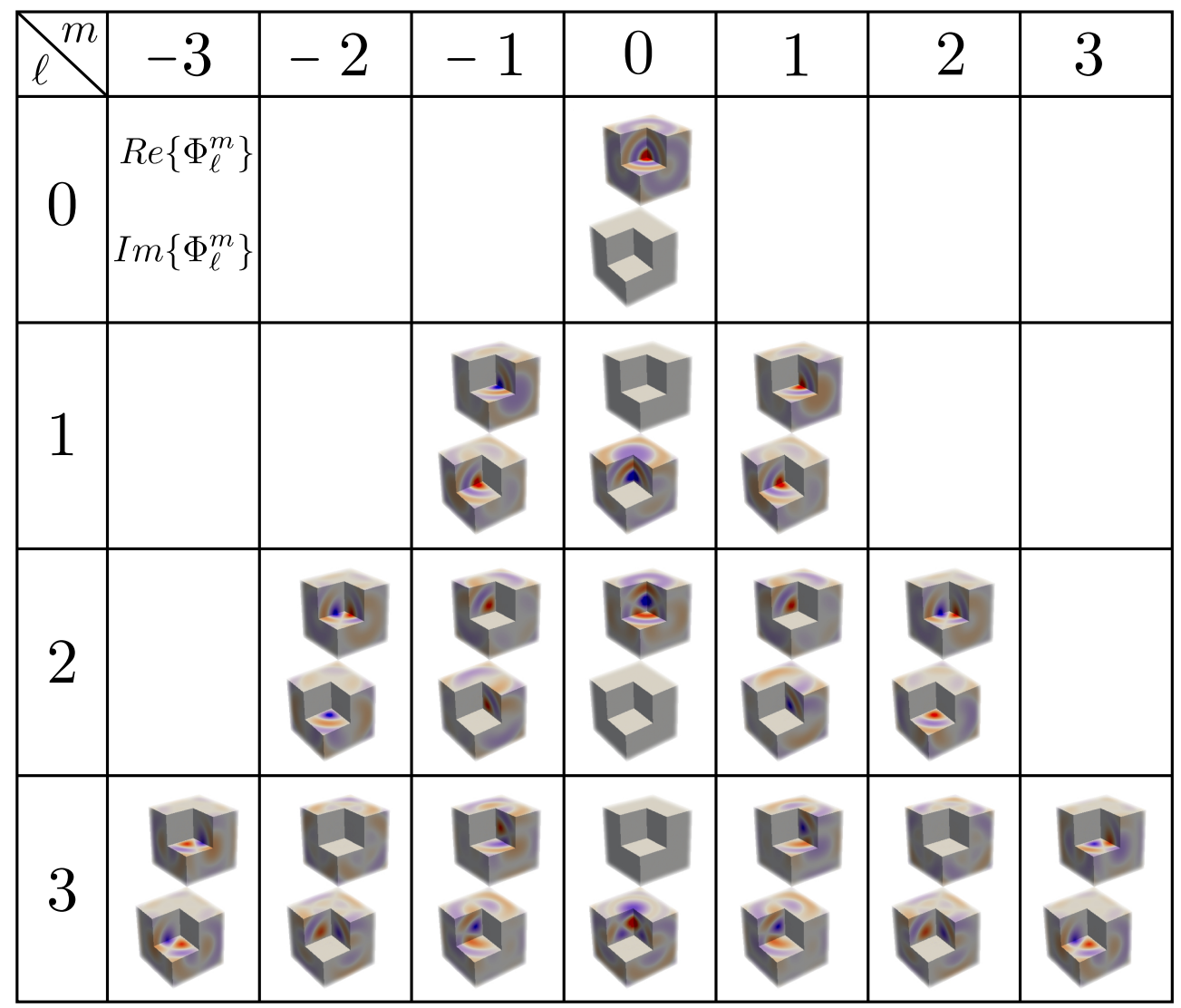

Figure 1. Real and imaginary parts of the shape functions $\Phi_{\ell}^{m}$ given by (14), for $0 \leq \ell \leq 3$.

functions $\Phi_{\ell}^{m}(\mathbf{x})$ is depicted for $0 \leq \ell \leq 3$ in Figure 1. Then, from (9), (10), (11) and (14), the pressure field is given at any point of $\Omega_{E}$ by

$$
p_{E}^{0}(\mathbf{x})=\sum_{\ell=0}^{N_{E}} \sum_{m=-\ell}^{\ell} \Phi_{\ell}^{m}(\mathbf{x}) a_{\ell m}^{E}=\sum_{\ell=0}^{N_{E}} \sum_{m=-\ell}^{\ell} 4 \pi i^{\ell} j_{\ell}\left(k r_{x}\right) Y_{\ell}^{m}\left(\theta_{x}, \varphi_{x}\right) a_{\ell m}^{E} \quad \mathbf{x} \in \Omega_{E}
$$

Note that without the analytical result (14), one would need to perform for each evaluation point $\mathbf{x}$ and each pair of indices $(\ell, m)$ a numerical quadrature over $\mathcal{C}$ requiring $O\left(N_{E}^{2}\right)$ quadrature points, adding considerable computational cost to the overall discrete VTCR treatment. The computational work required for evaluating the pressure field is thus clearly proportional to the number $N_{\text {eval }}$ of evaluation points $\mathbf{x}$.

\subsection{Discrete VTCR formulation}

Once a discretization of type (10), (11) has been chosen for each cavity, the weak formulation (4) leads to a complex-valued system of linear equations:

$$
\mathbb{K} \mathbb{A}=\mathbb{F}
$$


where the vector $\mathbb{A}=\left\{a_{\ell m}^{E}, 0 \leq \ell \leq N_{E},-\ell \leq m \leq \ell, 1 \leq E \leq N_{\Omega}\right\}$ collects all the unknown amplitude coefficients, the influence matrix $\mathbb{K}$ corresponds to the discretization of the bilinear terms of (4) and the load vector $\mathbb{F}$ results from all linear terms of (4). The influence matrix $\mathbb{K}$ is not symmetric and may be partitioned into $N_{\Omega}^{2}$ blocks $\mathbb{K}_{E E^{\prime}}$. Blocks $\mathbb{K}_{E E^{\prime}}$ are zero unless the subcavities $\Omega_{E}$ and $\Omega_{E^{\prime}}$ share a common interface $\Gamma_{E E^{\prime}}$ (this includes the case $E=E^{\prime}$ ). All nonzero blocks $\mathbb{K}_{E E^{\prime}}$ are fully populated since the shape functions are defined over entire acoustic sub-cavities $\Omega_{E}$. Letting $N$ denote the total number of DOFs of the VTCR problem (i.e. the overall number of unknown coefficients $\left.a_{\ell m}^{E}\right)$, one thus has $\mathbb{K} \in \mathbb{C}^{N, N}$ and $\mathbb{A}, \mathbb{F} \in \mathbb{C}^{N}$.

To exemplify the procedure underlying the set-up of system (16), consider the computation of a typical contribution, namely that arising in (4) from a face $\partial_{p} \Omega_{E}$ of a sub-cavity $\Omega_{E}$ on which a prescribed pressure $p_{d}$ is applied:

$$
\begin{gathered}
\int_{\partial_{p} \Omega_{E}}\left(\sum_{\ell=0}^{N_{E}} \sum_{m=-\ell}^{\ell} a_{\ell m}^{E} \Phi_{\ell}^{m}(\mathbf{x})-p_{d}\right)\left(\sum_{\ell^{\prime}=0}^{N_{E}} \sum_{m^{\prime}=-\ell^{\prime}}^{\ell^{\prime}} \overline{\delta a_{\ell^{\prime} m^{\prime}}^{E} L_{v}\left(\Phi_{\ell^{\prime}}^{m^{\prime}}(\mathbf{x})\right)}\right) \mathrm{d} S \\
=\sum_{\ell^{\prime}=0}^{N_{E}} \sum_{m^{\prime}=-\ell^{\prime}}^{\ell^{\prime}} \overline{\delta a_{\ell^{\prime} m^{\prime}}^{E}}\left\{\sum_{\ell=0}^{N_{E}} \sum_{m=-\ell}^{\ell}\left[\int_{\partial_{p} \Omega_{E}} \Phi_{\ell}^{m}(\mathbf{x}) \overline{L_{v}\left(\Phi_{\ell^{\prime}}^{m^{\prime}}(\mathbf{x})\right)} \mathrm{d} S\right] a_{\ell m}^{E}\right. \\
\left.-\int_{\partial_{p} \Omega_{E}} p_{d}(\mathbf{x}) \overline{L_{v}\left(\Phi_{\ell^{\prime}}^{m^{\prime}}(\mathbf{x})\right)} \mathrm{d} S\right\}
\end{gathered}
$$

The first and second integrals over $\partial_{p} \Omega_{E}$ in the right-hand side of (17) respectively contribute to the submatrix $\mathbb{K}_{E E}$ of the influence matrix $\mathbb{K}$ and the subvector $\mathbb{F}_{E}$ of the load vector $\mathbb{F}$.

As shown in section 3.2, the shape functions $\Phi_{\ell}^{m}(\mathbf{x})$ are evaluated analytically at any point $\mathbf{x}$. In expressions such as (17), the evaluation points $\mathbf{x}$ are quadrature points on relevant sub-cavity surfaces or interfaces. Moreover, the computation of $L_{v}\left(\Phi_{\ell}^{m}(\mathbf{x})\right)$ is straightforward by expressing $\nabla \Phi_{\ell}^{m}$ in spherical coordinates and using the following identities:

$$
\begin{aligned}
\frac{\partial}{\partial r} j_{\ell}(k r) & =-\frac{1}{2 r} j_{\ell}(k r)+\frac{k}{2} j_{\ell-1}(k r)-\frac{k}{2} j_{\ell+1}(k r) \\
\frac{\partial}{\partial \theta} Y_{\ell}^{m}(\theta, \varphi) & =m \cot \theta Y_{\ell}^{m}(\theta, \varphi)+e^{-i m \varphi} \sqrt{(\ell-m)(\ell+m+1)} Y_{\ell}^{m+1}(\theta, \varphi) \\
\frac{\partial}{\partial \varphi} Y_{\ell}^{m}(\theta, \varphi) & =\operatorname{im} Y_{\ell}^{m}(\theta, \varphi)
\end{aligned}
$$

Then, the integrals in (17) are computed using the following approach: (i) each surface (e.g. $\partial_{p} \Omega_{E}$ ) is meshed (for numerical quadrature purposes only) using linear triangular $C^{0}$ elements; (ii) The $\Phi_{\ell}^{m}$ and their derivatives are evaluated (using (14) and (18)) at the nodes; (iii) integrals are evaluated element-wise by means of usual FEM-type numerical quadrature. The quadrature mesh density must be adjusted to the acoustic wavelength. The number $N_{\text {int }}$ of quadrature elements per wavelength is thus another important computational parameter of the VTCR. Its effect will be discussed in the numerical examples of Sec. 4. Overall, 
setting up the system (16) only requires numerical quadratures over surfaces, making it easy to apply the VTCR to complex geometrical configurations.

\subsection{Adjusting the discretization parameter}

In the $2 \mathrm{D}$ case, the energy of the Fourier shape functions (8) decays in a known way as their order $|n|$ increases $^{27}$. This property was used to define an a priori selection criterion for the discretization parameter $N_{E}$ in each sub-cavity $\Omega_{E}$. This approach is now investigated for the 3D case, as the shape functions $\Phi_{\ell}^{m}$ are the natural 3D generalization of the Fourier shape functions (8). The energy $e(p ; \Omega)$ of a pressure field $p$ in a domain $\Omega$ is given by

$$
e(p ; \Omega):=\frac{1}{\rho c^{2}} \int_{\Omega} p(\mathbf{x}) \overline{p(\mathbf{x})} \mathrm{d} V(\mathbf{x})
$$

In particular, the energy of the shape function $\Phi_{\ell}^{m}$ is estimated, from expression (14), as

$$
\begin{aligned}
\rho c^{2} e\left(\Phi_{\ell}^{m} ; \Omega_{E}\right)=16 \pi^{2} \int_{\Omega_{E}}\left|j_{\ell}\left(k r_{x}\right)\right|^{2}\left|Y_{\ell}^{m}\left(\theta_{x}, \varphi_{x}\right)\right|^{2} \mathrm{~d} V(\mathbf{x}) \\
\leq 16 \pi^{2} \int_{0}^{R_{E}}\left|j_{\ell}\left(k r_{x}\right)\right|^{2} r_{x}^{2} \mathrm{~d} r_{x}=\frac{2 \pi^{2} R_{E}^{3}}{2 \ell+3}\left[\frac{e k R_{E}}{2 \ell}\right]^{2 \ell}\left(1+O\left(\frac{1}{\ell}\right)\right)
\end{aligned}
$$

(having invoked the orthogonality property (12)), where $R_{E}$ is the radius of the smallest ball centered at $\mathbf{x}$ containing $\Omega_{E}$. The last equality in (20) results from an analytical estimation of the radial integral, using the known large-order asymptotic behavior $j_{\ell}(z)=(2 \sqrt{2} \ell)^{-1}[e z / 2 \ell]^{2 \ell}\left(1+O\left(\ell^{-1}\right)\right)$ of $j_{\ell}$ (see e.g. identity 10.19 .1 of $^{33}$, and with $e=2.71828 \ldots$ denoting the base of the natural logarithm). The upper bound (20) shows that (i) $e\left(\Phi_{\ell}^{m} ; \Omega_{E}\right)$ has a faster-than-exponential decay as the order $\ell$ becomes arbitrarily large, and (ii) that the decay sets in for values of $\ell$ larger than $e k R_{E} / 2$, a threshold which is proportional to the characteristic radius of $\Omega_{E}$ measured in wavelengths. As an illustration, the energies $e\left(\Phi_{\ell}^{m} ; \Omega\right)$ are plotted in Figure 2 for a $1 \mathrm{~m} \times 1 \mathrm{~m} \times 1 \mathrm{~m}$ cubic cavity $\Omega$ filled with

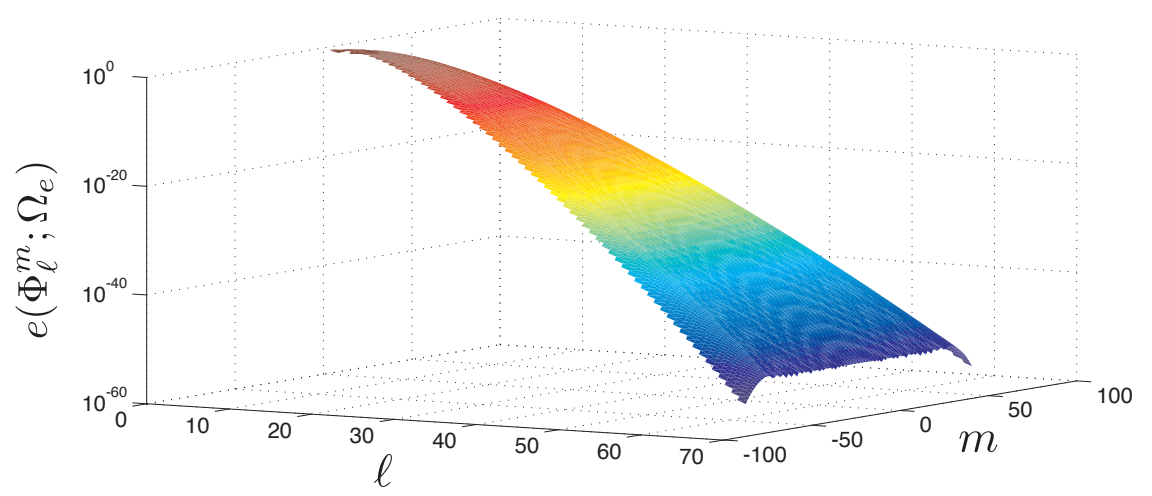

Figure 2. Energy $e\left(\Phi_{\ell}^{m} ; \Omega_{E}\right)$ of the 3-D VTCR shape functions versus its order $\ell$ and momentum $m$. 


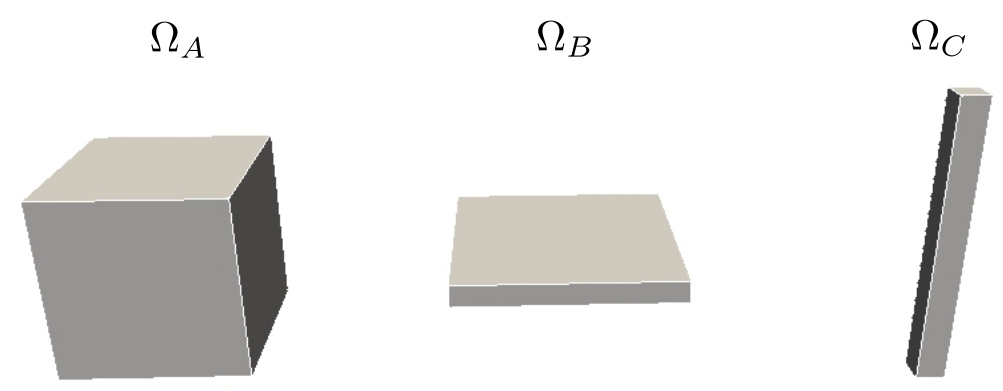

Figure 3. Convergence study (Section 3.4): geometry of box-shaped cavities $\Omega_{A}, \Omega_{B}$ and $\Omega_{C}$.

air at $1000 \mathrm{~Hz}$. Since it is plotted using a semilog scale, the observed concave shape along the $\ell$ direction of the surface corroborates the predicted faster-than-exponential asymptotic decay of $e\left(\Phi_{\ell}^{m} ; \Omega\right)$ with $\ell$.

The largest order $\ell=N_{E}$ in the definition (10) of the approximation space $S_{a d, 0}^{E, N_{E}}$ is then chosen according to

$$
\forall \ell \leqslant N_{E} \quad \max _{|m| \leqslant \ell} \frac{e\left(\Phi_{\ell}^{m} ; \Omega_{E}\right)}{e\left(\Phi_{0}^{0} ; \Omega_{E}\right)} \geqslant 10^{-r}
$$

(where $r$ is a given number related to the desired precision). The selection criterion (21) is the direct generalization of that previously proposed ${ }^{27}$ for $2 \mathrm{D}$ problems.

A sensible value for $\ell=N_{E}$ may alternatively be selected from a simple geometrical criterion, through

$$
N_{E}=2 \alpha k R_{E}
$$

where the desired accuracy is tuned by setting the non-dimensional coefficient $\alpha$. Note that $\alpha=e / 2$ corresponds to the onset of fast decay of $e\left(\Phi_{\ell}^{m} ; \Omega_{E}\right)$ predicted by the large-order asymptotic (20).

The convergence of the VTCR is studied in the following example, where criteria (21) and (22) are compared and suitable values for parameters $r$ and $\alpha$ sought. Let $\Omega_{A}, \Omega_{B}$ and $\Omega_{C}$ be three box-shaped cavities (Figure 3 ) with respective dimensions $a \times a \times a, b \times b \times(b / 10)$ and $(c / 10) \times(c / 10) \times c$, with the lengths $a, b, c$ (in meters) chosen so that all eight vertices of $\Omega_{A}, \Omega_{B}$ and $\Omega_{C}$ lie on a sphere of radius $R=1 \mathrm{~m}$. An impedance condition of type (1b) is applied over the whole cavity boundary $\partial \Omega_{A}, \partial \Omega_{B}$ or $\partial \Omega_{C}$, with $Z=845-50 i \mathrm{~Pa}^{-1}{ }^{-1}$.s. The loading $h_{d}(\mathbf{x})$ is defined so that the exact solution $p_{\text {ex }}$ is

$$
p_{e x}(\mathbf{x})=\sum_{\ell=0}^{N} \sum_{m=-\ell}^{\ell} s_{\ell m} \Phi_{\ell}^{m}(\mathbf{x})
$$

where the maximum order $N_{\text {ex }}$ is defined by $N_{\text {ex }}=\lfloor 20 k\rfloor,\left\{s_{\ell m}\right\}$ is a random vector of length $\left(N_{\text {ex }}+1\right)^{2}$, and the reference point $\mathbf{x}_{E}$ entering the shape functions $\Phi_{\ell}^{m}$ is randomly chosen inside the cavity. Five frequencies, such that $2 k R=10,20,30,40$ and 50, are considered 


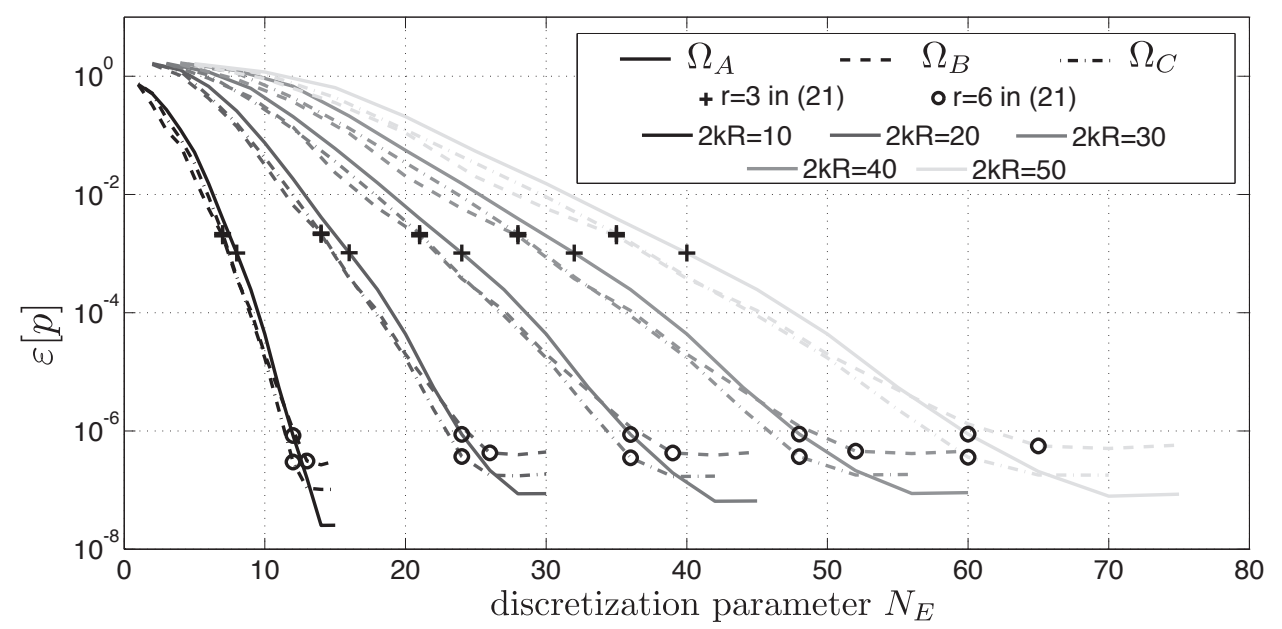

Figure 4. Convergence study (Section 3.4): Solution error $\varepsilon[p]$ (24) for the three geometries against the discretization parameter $N_{E}$, for several frequencies.

for each cavity shape. The convergence is evaluated using the relative solution error $\varepsilon[p]$ :

$$
\varepsilon[p]:=\frac{\left\|p-p_{e x}\right\|_{H^{1}(\Omega)}}{\left\|p_{e x}\right\|_{H^{1}(\Omega)}}
$$

Figure 4 plots the converged error (24) against $N_{E}$ for cavities A, B and C. For each frequency considered, the convergence trend is similar for the three cavities. Furthermore, $\varepsilon[p]=10^{-4}$ was found to be achieved in all cases by setting $\alpha=1$ in (22). Indeed, $\varepsilon[p]$ is slightly lower than $10^{-4}$ in all cases with $N_{E}=2 k R$; this corresponds to setting $\alpha=1$ in (22) (this is consistent with the previously-mentioned, somewhat larger but comparable, value $\alpha=e / 2$ for the onset of rapid decrease of $e\left(\Phi_{\ell}^{m}, \Omega\right)$. In addition, Figure 4 indicates the value of $N_{E}$ given by the energy-based criterion (21) with $r=3$ (crosses) and $r=6$ (circles). Criterion (21) is thus seen to also provide a consistent estimation of $N_{E}$ for varying frequency and geometry, with achieved solution errors $\varepsilon[p]$ very similar for all the considered cases. From these results one may conclude that both criteria (21) and (22) yield good $a$ priori estimates for $N_{E}$.

\subsection{Conditioning issues and rescaling of VTCR system}

The VTCR, like other numerical strategies that use oscillating functions in the approximation spaces, suffers from ill-conditioning (see for example ${ }^{17,11,25}$ ). Then, as with the $2 \mathrm{D}$ case $^{27}$, a preconditioner is used to reduce the effect of ill-conditioning. It is defined as the diagonal matrix $\mathbb{P}:=\operatorname{Diag}\left(\left[e\left(\Phi_{\ell}^{m} ; \Omega_{E}\right)\right]^{-1 / 2}\right)$, and amounts to set up the VTCR system (16) using energy-normalized shape functions, i.e. to replace the representation (15) of the pres- 
sure inside a sub-cavity by

$$
p_{E}^{0}(\mathbf{x})=\sum_{\ell=0}^{N_{E}} \sum_{m=-\ell}^{\ell} \widehat{\Phi}_{\ell}^{m}(\mathbf{x}) \widehat{a}_{\ell m}^{E}
$$

where the energy-normalized shape functions and rescaled coefficients are defined by

$$
\text { (a) } \widehat{\Phi}_{\ell}^{m}(\mathbf{x}):=\left[e\left(\Phi_{\ell}^{m} ; \Omega_{E}\right)\right]^{-1 / 2} \Phi_{\ell}^{m}(\mathbf{x}), \quad \text { (b) } \widehat{a}_{\ell m}^{E}:=\left[e\left(\Phi_{\ell}^{m} ; \Omega_{E}\right)\right]^{1 / 2} a_{\ell m}^{E}
$$

The scaled version $\widehat{\mathbb{K}} \widehat{\mathbb{A}}=\widehat{\mathbb{F}}$ of the system (16) (where $\widehat{\mathbb{K}}=\mathbb{P} \mathbb{K} \mathbb{P}, \widehat{\mathbb{F}}=\mathbb{P F}$ and $\widehat{\mathbb{A}}=\mathbb{P}^{-1} \mathbb{A}$ is then solved using the Moore-Penrose pseudo-inverse, an approach which was found to perform satisfactorily despite the residual ill-conditioning of the rescaled matrix $\widehat{\mathbb{K}}$.

\subsection{Rescaled amplitude portrait: its interpretation and convergence}

In addition to improving the condition number of the VTCR system, the rescaled amplitude portrait defined in terms of modified coefficients (25b) is now shown to allow estimating the pressure directivity inside a sub-cavity. Indeed, using (14), the representation (25) becomes

$$
p_{E}^{0}(\mathbf{x})=\sum_{\ell=0}^{N_{E}} \sum_{m=-\ell}^{\ell} \phi_{\ell}\left(k r_{x}\right) Y_{\ell}^{m}\left(\theta_{x}, \varphi_{x}\right) \widehat{a}_{\ell m}^{E}
$$

with $\phi_{\ell}(z):=4 \pi \mathrm{i}^{\ell}\left[e\left(\Phi_{\ell}^{m} ; \Omega_{E}\right)\right]^{-1 / 2} j_{\ell}(z)$. Now, since $\phi_{\ell}\left(k r_{x}\right) Y_{\ell}^{m}\left(\theta_{x}, \varphi_{x}\right)=\widehat{\Phi}_{\ell}^{m}(\mathbf{x})$ and due to the $L^{2}$-orthonormality of the $Y_{\ell}^{m}$ on $\mathcal{C}$, one has

$$
\int_{0}^{R_{E}}\left|\phi_{\ell}\left(k r_{x}\right)\right|^{2} r_{x}^{2} \mathrm{~d} r=1
$$

for any spherical sub-cavity (with $R_{E}$ denoting its radius). For non-spherical sub-cavities (e.g. star-shaped sub-cavities with the upper integration bound $R_{E}$ now depending on $\left.\left(\theta_{x}, \varphi_{x}\right)\right)$, the above integral is still $O(1)$ for any $\ell$. From this remark, the rescaled amplitude portrait $\widehat{A}_{E}$ defined by

$$
\widehat{A}_{E}\left(\theta_{x}, \varphi_{x}\right):=\sum_{\ell=0}^{N_{E}} \sum_{m=-\ell}^{\ell} Y_{\ell}^{m}\left(\theta_{x}, \varphi_{x}\right) \widehat{a}_{\ell m}^{E}
$$

is expected to reflect the pressure directivity inside $\Omega_{E}$, and in particular to reveal the directions along which most of the energy propagates. Clearly, $\widehat{A}_{E}$ describes the pressure directivity more accurately than the original portrait $A_{E}$ because the coefficients of the latter appear, in representation (15), as factors of non-normalized (rather than normalized) shape functions.

A directly related consideration is the fact that the convergence of $p_{E}^{0}$ in the $L^{2}\left(\Omega_{E}\right)$ norm as $N_{E} \rightarrow \infty$ does not imply the convergence of the amplitude portrait $A_{E}(\theta, \varphi)$ in the $L^{2}(\mathcal{C})$ norm. To illustrate this behavior, consider the case where $A_{E}(\theta, \varphi)$ is perturbed by a spurious additional term, resulting in a new distribution $\tilde{A}_{E}(\theta, \varphi)$ such that

$$
\tilde{A}_{E}(\theta, \varphi)=A_{E}(\theta, \varphi)+\alpha Y_{\ell}^{m}(\theta, \varphi)
$$


with $\alpha$ being the (finite but not necessarily small) perturbation amplitude. Let $p_{E}=\mathcal{H}\left[A_{E}\right]$ and $\tilde{p}_{E}=\mathcal{H}\left[\tilde{A}_{E}\right]$ denote the corresponding pressure fields, see (5). One then has (using orthonormality (12) of $\left.Y_{\ell}^{m}\right)$ :

$$
\left\|A_{E}-\tilde{A}_{E}\right\|_{L^{2}(\mathcal{C})}^{2}=|\alpha|^{2}
$$

and hence

$$
\left\|\widehat{A}_{E}-\widehat{\tilde{A}}_{E}\right\|_{L^{2}(\mathcal{C})}^{2}=|\widehat{\alpha}|^{2},
$$

where $\widehat{\alpha}$ is the rescaled perturbation amplitude given by (26b), while (see (5), (10) and (19))

$$
\left\|p_{E}-\tilde{p}_{E}\right\|_{L^{2}\left(\Omega_{E}\right)}^{2}=|\alpha|^{2}\left\|\mathcal{H}\left[Y_{\ell}^{m}\right]\right\|_{L^{2}\left(\Omega_{E}\right)}^{2}=|\alpha|^{2}\left\|\Phi_{\ell}^{m}\right\|_{L^{2}\left(\Omega_{E}\right)}^{2}=|\alpha|^{2} e\left(\Phi_{\ell}^{m} ; \Omega_{E}\right)=|\widehat{\alpha}|^{2} .
$$

For finite $\alpha$ but increasing $\ell$, and due to the decay property (20) of $e\left(\Phi_{\ell}^{m} ; \Omega_{E}\right)$, one has $\left\|p_{E}-\tilde{p}_{E}\right\|_{L^{2}\left(\Omega_{E}\right)}^{2} \rightarrow 0$ even though $\left\|A_{E}-\tilde{A}_{E}\right\|_{L^{2}(\mathcal{C})}^{2} \nrightarrow \rightarrow 0$ as $\ell \rightarrow \infty$. In contrast, one

simultaneously has $\left\|p_{E}-\tilde{p}_{E}\right\|_{L^{2}\left(\Omega_{E}\right)}^{2} \rightarrow 0$ and $\left\|\widehat{A}_{E}-\widehat{\tilde{A}}_{E}\right\|_{L^{2}(\mathcal{C})}^{2} \rightarrow 0$ as $\ell \rightarrow \infty$, emphasizing that the convergence on the solution $p_{E}$ is correlated to that of the rescaled, rather than original, amplitude portrait. In practice, while the amplitude portrait $A_{E}$ (and especially coefficients $\alpha_{\ell m}^{E}$ for high order $n$ ) may be sensitive to data perturbations, no stability issues arose in numerical experiments for the evaluation of $p_{E}$. Hence, unlike in some other methods such as discontinuous Galerkin formulations, no stabilization strategy (such as additional penalty terms) is implemented in the present form of the VTCR.

\section{Numerical results}

This section presents a set of numerical examples that were designed to study the performance of the 3D acoustic VTCR formulation (Secs. 4.1 and 4.2), followed by an actual case study (three-dimensional car cavity, Sec. 4.3). All results to follow were obtained on a MacBook with a core 2 duo $2.4 \mathrm{Ghz}$ CPU and a 4B RAM.

\subsection{Cubic cavity}

First, let us consider a simple cavity problem having a known exact solution. The cavity domain $\Omega$ is the cube $\left\{\left(x_{1}, x_{2}, x_{3}\right) \in[0, L] \times[0, L] \times[0, L]\right\}$, and an impedance boundary condition of type (1b) is assumed over $\partial \Omega$ with $Z, h_{d}$ chosen such that the exact solution $p_{e x}$ is a plane wave propagating in the oblique spherical direction $\left(\theta_{e x}=0.8, \varphi_{e x}=2.3\right)$. The cavity $\Omega$ is taken as a whole, i.e. is not subdivided into sub-cavities. The convergence with respect to the dimension of the approximation space $S_{a d, 0}^{E, N_{E}}$ (see (10) and (21)) and the density $N_{\text {int }}$ of the surface quadrature mesh (see Sec. 3.3) is evaluated for four frequencies, corresponding to $k L=5,20,35$ and 50, and three quadrature densities $N_{\text {int }}=7,17$ and 27 . The relative solution errors $\varepsilon[p]$ (see (24)) are reported on Figure 5 .

For each considered frequency, $N_{E}$ (and hence the problem size $\left.\left(N_{E}+1\right)^{2}\right)$ is set using criterion (21) with $r$ varying up to 8 . The 3D VTCR exhibits a very good convergence, with a rapid decrease towards very small values of the solution error as $r$ is increased. Moreover, 


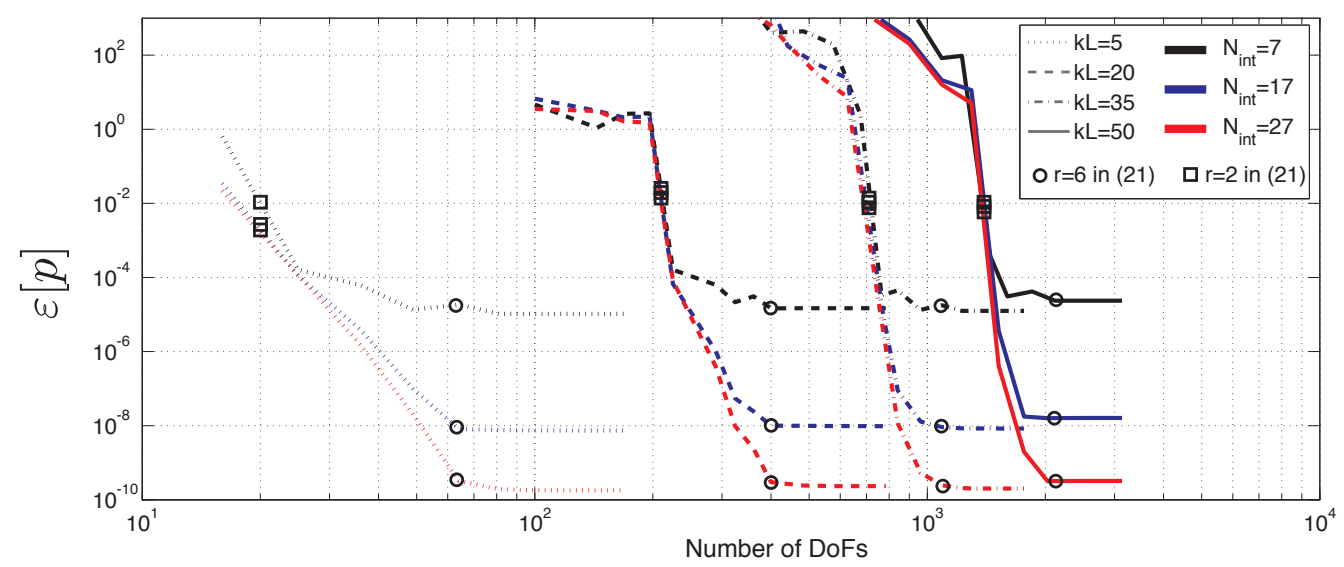

Figure 5. Cubic cavity (Section 4.1): convergence of the 3-D VTCR for the pressure field evaluation, with $k L=5, k L=20, k L=35$ and $k L=50$

even small values of $r$ in criterion (21) yield an acceptable accuracy. For example, with $r=2$ (which corresponds to the square symbol on each curve), the solution errors are already down to around $1 \%$.

Figure 5 also shows that convergence is strongly influenced by the quadrature density $N_{\text {int }}$. Non-uniform convergence is seen to occur for the coarsest quadrature density $N_{\text {int }}$. Moreover, residual quadrature error prevents accuracy to improve beyond a certain refinement level, which depends on the chosen value of $N_{\text {int }}$. While high solution accuracy requires a refined surface quadrature mesh (with significant impact on the setup CPU time), one also observes that the relatively coarse quadrature density $N_{\text {int }}=7$ is suffcient for reaching solution accuracies of the order of $1 \%$, sufficient for many engineering applications.

The CPU times required to (i) set up the system (16), (ii) solve it, and (iii) evaluate the pressure inside the cavity using (15), respectively denoted $T_{\text {setup }}, T_{\text {solve }}$ and $T_{\text {post }}$, are summarized in Table 1 for the values of $k L$ and $N_{\text {int }}$ considered, the problem sizes $N$ corresponding to $N_{E}$ set using $r=6$ in (21) (the solution errors for this case being indicated

Table 1. Cubic cavity (Section 4.1): CPU times required to set up system (16), solve it, and evaluate $p$ inside the cavity using (15)

\begin{tabular}{|c|c|c|c|c|c|c|c|c|c|}
\hline$k L$ & \multicolumn{3}{|c|}{5} & \multicolumn{3}{c|}{20} & \multicolumn{3}{c|}{50} \\
\hline$N_{E}$ & \multicolumn{3}{|c|}{64} & \multicolumn{3}{c|}{400} & \multicolumn{3}{c|}{2116} \\
\hline$N_{\text {int }}$ & 7 & 17 & 27 & 7 & 17 & 27 & 7 & 17 & 27 \\
\hline$T_{\text {setup }}(\mathrm{s})$ & 0.18 & 0.36 & 0.78 & 12 & 62 & 217 & 332 & 3250 & 11380 \\
\hline$T_{\text {solve }}(\mathrm{s})$ & \multicolumn{3}{|c|}{$3 \times 10^{-4}$} & \multicolumn{3}{c|}{0.02} & \multicolumn{3}{c|}{0.8} \\
\hline$N_{\text {eval }}$ & \multicolumn{3}{|c|}{265} & \multicolumn{3}{c|}{2428} & \multicolumn{3}{c|}{39983} \\
\hline$T_{\text {post }}(\mathrm{s})$ & \multicolumn{3}{|c|}{0.03} & \multicolumn{3}{c|}{73} \\
\hline
\end{tabular}




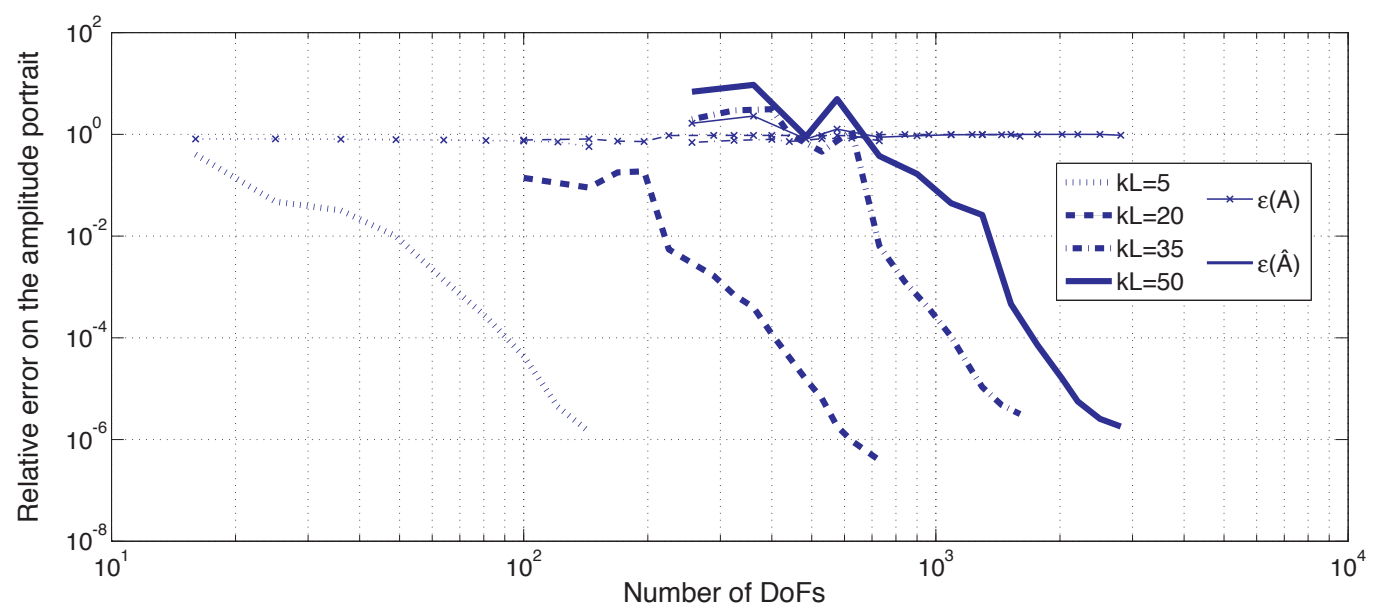

Figure 6. Cubic cavity (Section 4.1): convergence on the rescaled (bold lines) and original (fine lines) amplitude portraits.

by a circle on Figure 5). The pressure field is evaluated at $N_{\text {eval }}$ points, the chosen values of $N_{\text {eval }}$ corresponding to about five points per wavelength for cases $k L=20$ and 50 .

Most of the computation time is consumed in setting up system (16), a step whose cost is moreover strongly influenced, not surprisingly, by the quadrature density $N_{\text {int }}$. The very high value of $T_{\text {setup }}$ observed in the $\left(k L, N_{\text {int }}\right)=(50,27)$ case is partly due to RAM memory limitations of the computer used. The solution times are comparatively negligible, implying that the non-symmetric character of the VTCR formulation only marginally affects its overall performance.

The relative $L^{2}$ errors on both the original amplitude portrait $A_{E}$ and its rescaled counterpart $\widehat{A}_{E}$ introduced in Sec. 3.6, denoted by $\varepsilon[A]$ and $\varepsilon[\widehat{A}]$, are reported on Figure 6 . Clearly, as anticipated in Sec. 3.6, this is a situation where $\widehat{A}_{E}$ converges as $N_{E} \rightarrow \infty$ while $A_{E}$ does not. Figure 7 shows the 3 -D representation of the rescaled amplitude portrait for the four considered frequencies. The main direction of propagation indicated by this representation corresponds to that of the exact solution, confirming the usefulness of $\widehat{A}_{E}$ for physically interpreting the VTCR solution.

\subsection{Scattering by a sound-hard sphere}

The scattering of a plane wave by a sound-hard spherical obstacle, bounded by a sphere $S_{1}$ of radius $R_{1}=0.5 \mathrm{~m}$, is now considered. The surrounding acoustic medium $\Omega$ is truncated by a concentric spherical surface $S_{2}$ of radius $R_{2}=1.8 \mathrm{~m}$. The problem (1) is solved with the following boundary conditions: (i) $L_{v}\left(p+p_{\text {scat }}\right)=0$ on $S_{1}$ (i.e. $\partial_{v} \Omega=S_{1}$ ) with $p_{\text {scat }}$ denoting a plane wave propagating in the spherical direction $(\theta, \varphi)=(\pi / 2,0)$, and (ii) $p+\rho_{0} c_{0} L_{v}(p)=0$ on $S_{2}$ (i.e. $\partial_{Z} \Omega=S_{2}$ ). The latter condition is an absorbing condition that approximates the Sommerfeld radiation condition. The domain $\Omega$ is decomposed into 32 sub-cavities as illustrated in Figure 8. Solution errors $\varepsilon[p]$ are evaluated with a multi- 
18
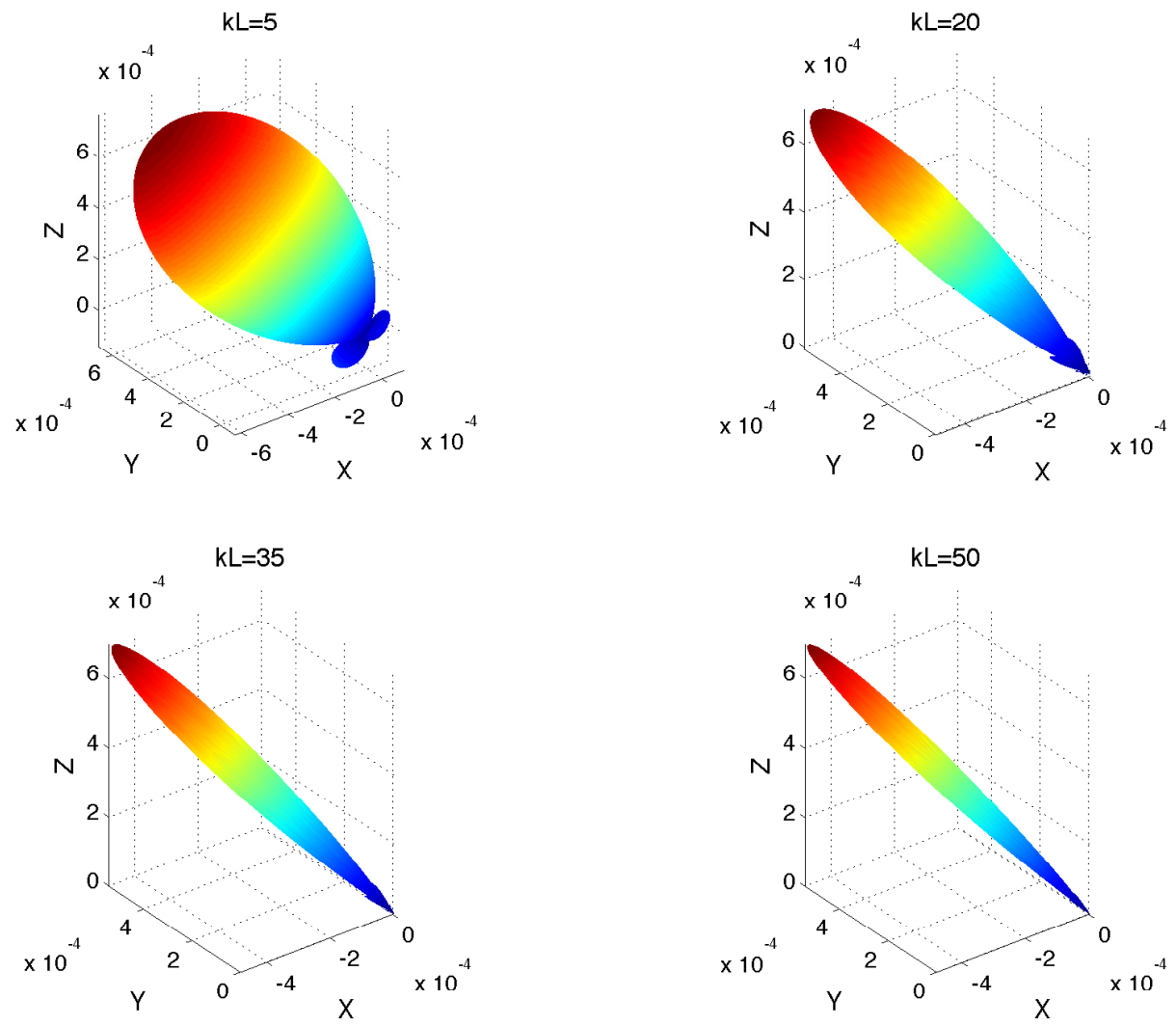

Figure 7. Cubic cavity (Section 4.1): rescaled amplitude portraits, $k L=5$ (top left), $k L=20$ (top right), $k L=35$ (bottom left) and $k L=50$ (bottom right).

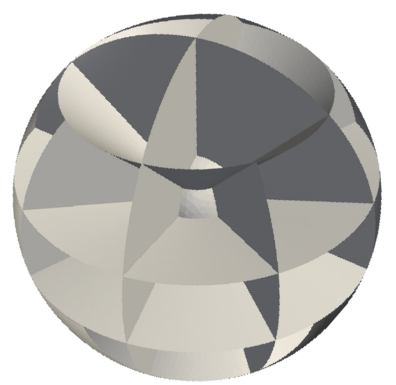

Figure 8. Scattering by a sound-hard sphere (Section 4.2): discretized computational domain.

cavity version of (24) which incorporates contributions from pressure discontinuities across interfaces $\Gamma_{E E^{\prime}}$ :

$$
\varepsilon[p]:=\frac{1}{\left\|p_{e x}\right\|_{H^{1}(\Omega)}}\left[\sum_{E=1}^{N_{\Omega}}\left\|p_{E}-p_{e x}\right\|_{H^{1}\left(\Omega_{E}\right)}^{2}+\sum_{E^{\prime}<E}\left\|p_{E}-p_{E^{\prime}}\right\|_{L^{2}\left(\Gamma_{E E^{\prime}}\right)}^{2}\right]^{1 / 2}
$$


Using separation of variables in spherical coordinates, the exact solution of the resulting problem is known to have the analytical form

$$
p^{e x}(r, \theta, \varphi)=\sum_{n=0}^{\infty} a_{n}(\theta, \varphi) h_{n}^{(1)}(k r)+b_{n}(\theta, \varphi) h_{n}^{(2)}(k r)
$$

where $h_{n}^{(1)}$ and $h_{n}^{(2)}$ are the spherical Hankel functions of the first and second kind, respectively ${ }^{33}$. Coefficients $a_{n}(\theta, \varphi)$ and $b_{n}(\theta, \varphi)$ are obtained by solving the system:

$$
\begin{aligned}
{\left[\begin{array}{cc}
h_{n}^{(1) \prime}\left(k R_{1}\right) & h_{n}^{(2) \prime}\left(k R_{1}\right) \\
h_{n}^{(1) \prime}\left(k R_{2}\right)-\mathrm{i} k h_{n}^{(1)}\left(k R_{2}\right) & h_{n}^{(2) \prime}\left(k R_{2}\right)-\mathrm{i} k h_{n}^{(2)}\left(k R_{2}\right)
\end{array}\right]\left\{\begin{array}{c}
a_{n}(\theta, \varphi) \\
b_{n}(\theta, \varphi)
\end{array}\right\} } & \\
& =\left\{\begin{array}{c}
-(2 n+1) \mathrm{i}^{n} k P_{n}(\cos \theta) j_{n}^{\prime}\left(k R_{1}\right) \\
0
\end{array}\right\}
\end{aligned}
$$

where $j_{n}$ is the spherical Bessel function of the first kind and $P_{n}$ the $n^{\text {th }}$ Legendre polynomial. Three circular frequencies are considered, such that $2 k R_{1}=5,20$ and 35 .

The real part of the scattered field is plotted in Fig. 9. The solution is consistent with physical intuition; one can for instance clearly see the reverberated and transmitted waves around the sphere.

Figure 10 reports the observed solution errors (as defined by (33)) against the total number $N$ of DOFs. Like in Fig. 5, each error curve corresponds to a sequence of problem sizes $N$ resulting from $N_{E}$ chosen using criterion (21) with $r$ varying from 1 to 6 , with a quadrature density $N_{\text {int }}=12$ used in all cases. Again, convergence is observed for the three considered frequencies. Convergence is however seen to be slower for the lowest frequency (for which the diameter of the sub-cavities are less than one wavelength), indicating that the VTCR indeed performs best in the mid-frequency range which motivated its design. The problem sizes yielding a relative solution error $\varepsilon[p] \approx 10^{-2}$ for the three considered
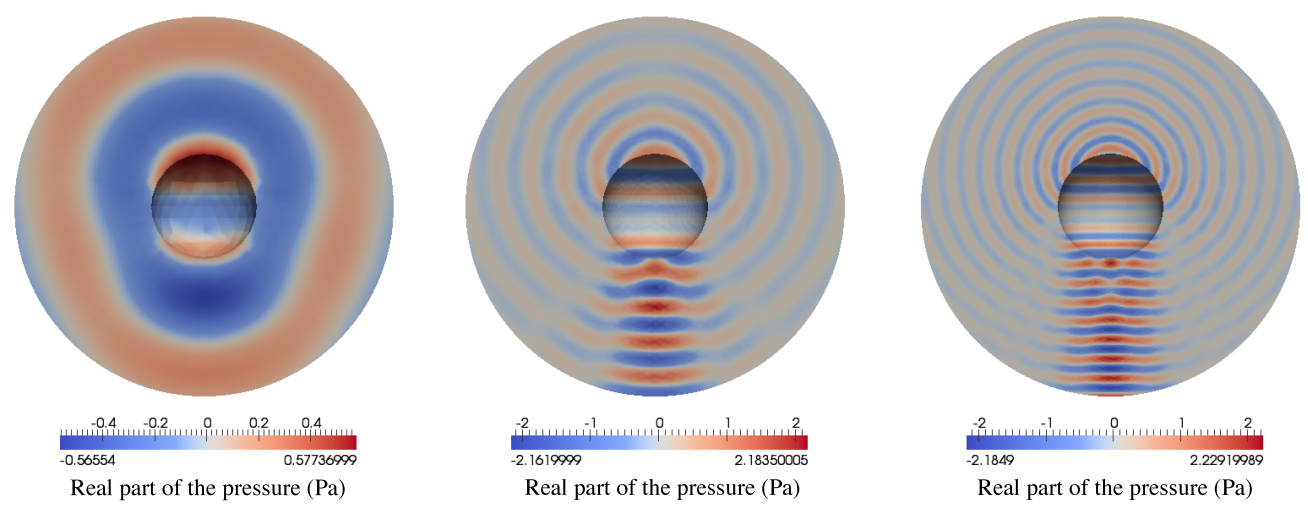

Figure 9. Scattering by a sound-hard sphere (Section 4.2): real part of the scattered pressure field for $2 k R_{1}=5$ (left), $2 k R_{1}=20$ (middle) and $2 k R_{1}=35$ (right) 


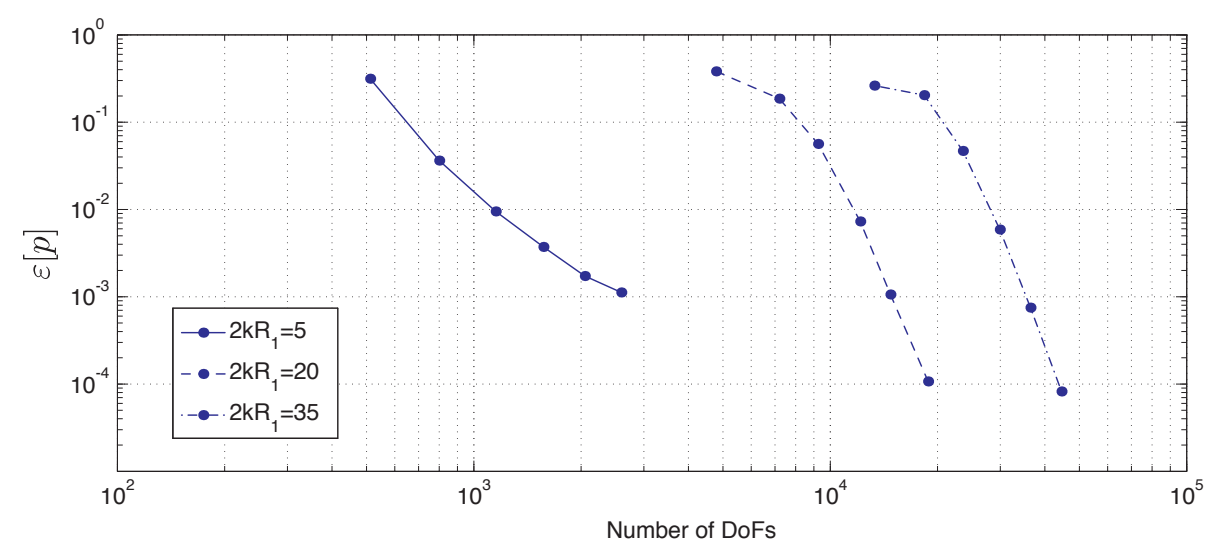

Figure 10. Scattering by a sound-hard sphere (Section 4.2): convergence curves for the relative solution error $\varepsilon[p](\operatorname{see}(33))$.

frequencies are $N=1152,12137$ and 29902, respectively obtained with $r=3,4$ and 4 in (21). Note that satisfactory reconstruction errors and convergences are achieved even though this example involves non-convex sub-cavities. Using this discretization, the CPU times incurred by the VTCR (in seconds) were $\left(T_{\text {setup }}, T_{\text {solve }}, T_{\text {post }}\right)=(5.5,0.1,0.23)$ for $2 k R_{1}=5,\left(T_{\text {setup }}, T_{\text {solve }}, T_{\text {post }}\right)=(719,27,79)$ for $2 k R_{1}=20$, and $\left(T_{\text {setup }}, T_{\text {solve }}, T_{\text {post }}\right)=$ $(4209,353,1189)$ for $2 k R_{1}=35$ (using the notations of Table 1). Again, most of the CPU time was consumed in the setup phase, and the performance for the largest model was hindered by insufficient RAM on the used platform. A FEM mesh for the same configuration and using only seven elements per wavelength would require approximately 30500, 130900 or 689800 nodes, respectively.

Finally, Fig. 11 shows the rescaled amplitude portraits at points located on either side
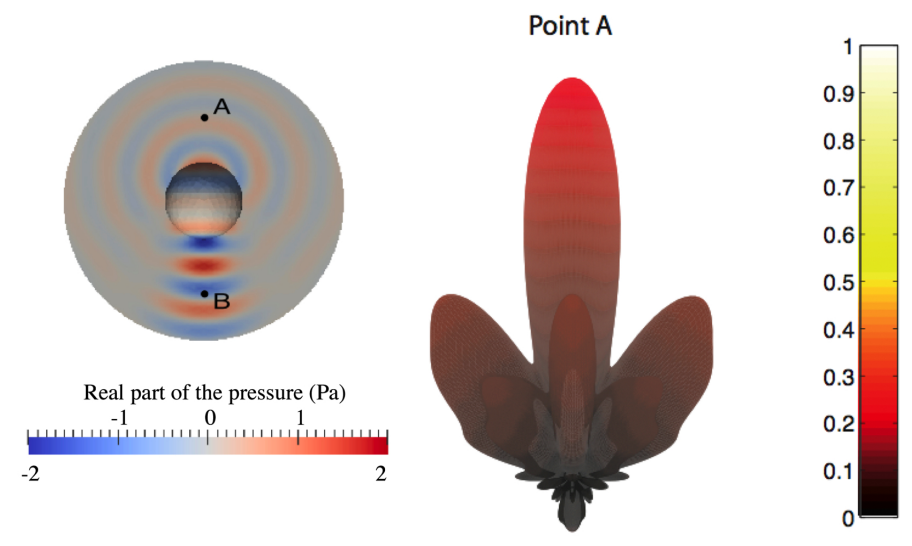

Figure 11. Scattering by a sound-hard sphere (Section 4.2): rescaled amplitude portraits computed at points A (middle) and B (right) defined in the left part

$$
\text { , for } 2 k R_{1}=12 \text {. }
$$


of the obstacle at $2 k R_{1}=12$. Again this representation correctly reveals the main direction of propagation.

\subsection{Three-dimensional car cavity}

Finally, the 3D acoustic VTCR is used to solve an acoustic problem for a car cavity (Fig. 12). The cavity, filled with air $\left(\rho=1.2 \mathrm{~kg} \cdot \mathrm{m}^{-3}, c=344 \mathrm{~m} \cdot \mathrm{s}^{-1}\right)$, is excited by a point source located in the front right part of the cavity. The front and rear windows are hard walls (boundary condition of type (1d) with $v_{d}=0$ ), while an impedance condition of type (1b) is prescribed over all other boundaries (with $Z=845-55 \mathrm{i} \mathrm{Pasm}^{-1}$ on the seats and $Z=615.4-1887 \mathrm{i} \mathrm{Pas} \mathrm{m}^{-1}$ elsewhere). The cavity is decomposed into 24 sub-cavities (see the right part of Fig. 12).

The pressure field computed by the VTCR for frequencies 700, 1200 and $1700 \mathrm{~Hz}$ is

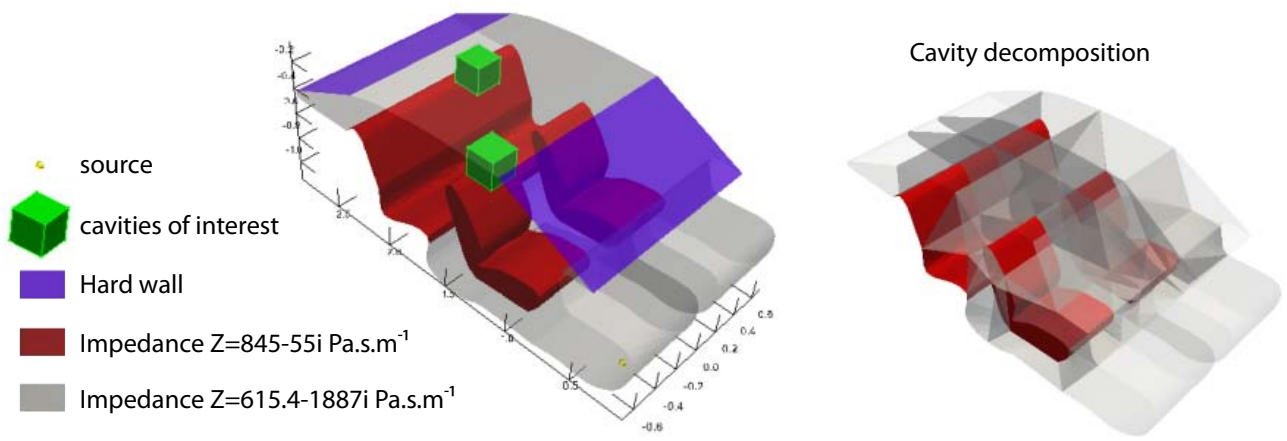

Figure 12. Definition of the car cavity of section 4.3 (boundary conditions on the left and decomposition into sub-cavities on the right).

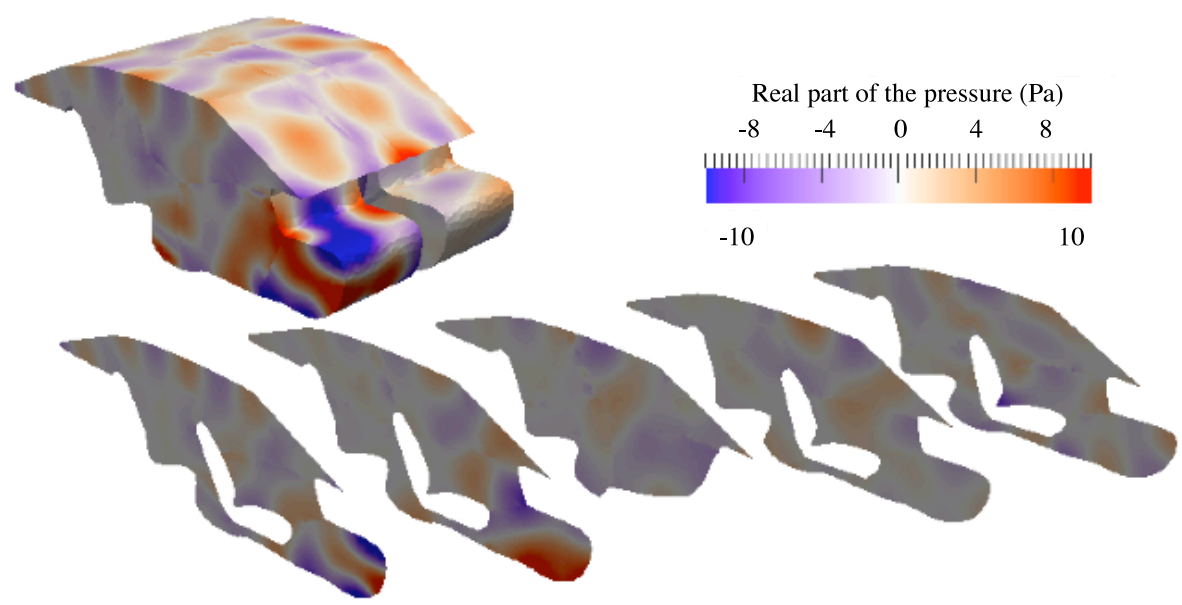

Figure 13. Real part of the pressure field computed with the 3-D VTRC in the problem defined in Figure 12 at $700 \mathrm{~Hz}$. 


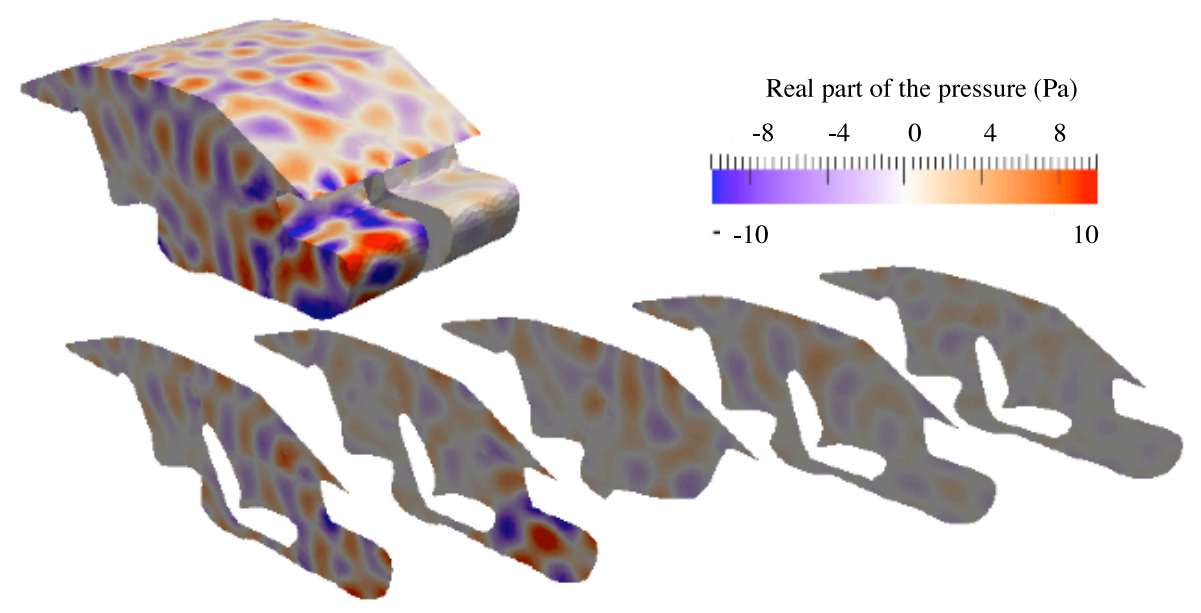

Figure 14. Real part of the pressure field computed with the 3-D VTRC in the problem defined in Figure 12 at $1200 \mathrm{~Hz}$.

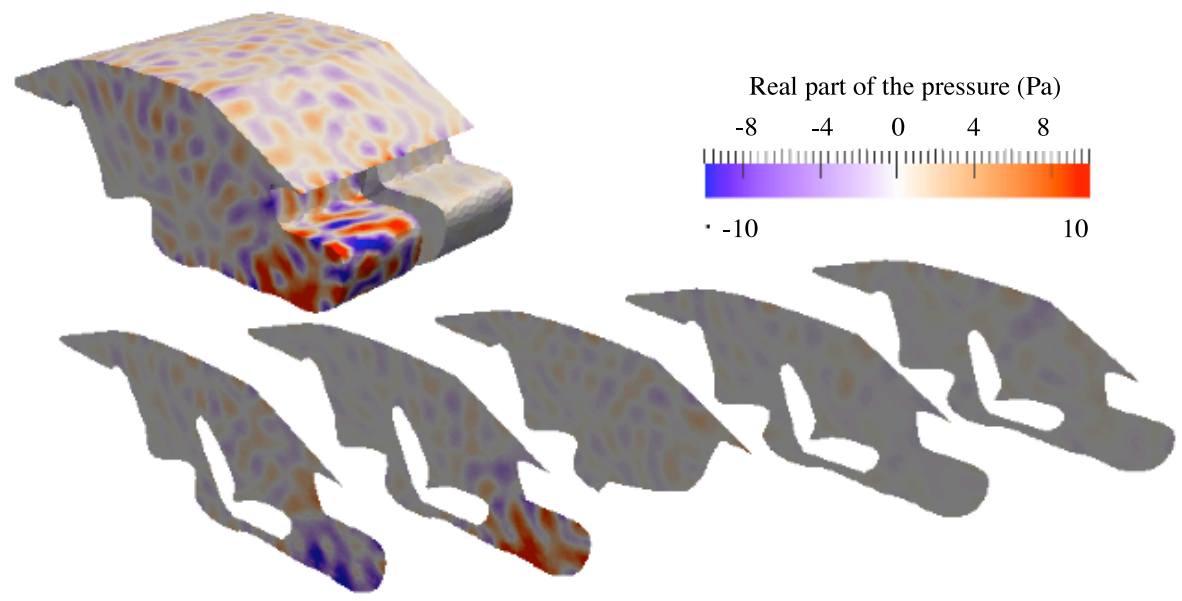

Figure 15. Real part of the pressure field computed with the 3-D VTRC in the problem defined in Figure 12 at $1700 \mathrm{~Hz}$.

plotted on Figures 13, 14 and 15, respectively. Setting up and solving the system (16) required about 2 and 45 minutes of CPU time, respectively, for the $700 \mathrm{~Hz}$ and $1700 \mathrm{~Hz}$ cases, with a quadrature density $N_{\text {int }} \approx 7$. The pressure in the whole car cavity is represented by means of several planar sections. The discretization parameters $N_{E}$ have been adjusted using (21) with $r=3$, leading to $N=4468,9034$ and 16342 DOFs overall, respectively.

The ability of the VTCR to treat complex 3D configurations may then be exploited for evaluating quantities of industrial interest. For example, the RMS average pressure evaluated in two selected cavities of interest (shown in Fig. 12), corresponding to cubic zones of 0.2 $\mathrm{m}$ length where the sound "ambiance" near the head of the front and rear passengers is 

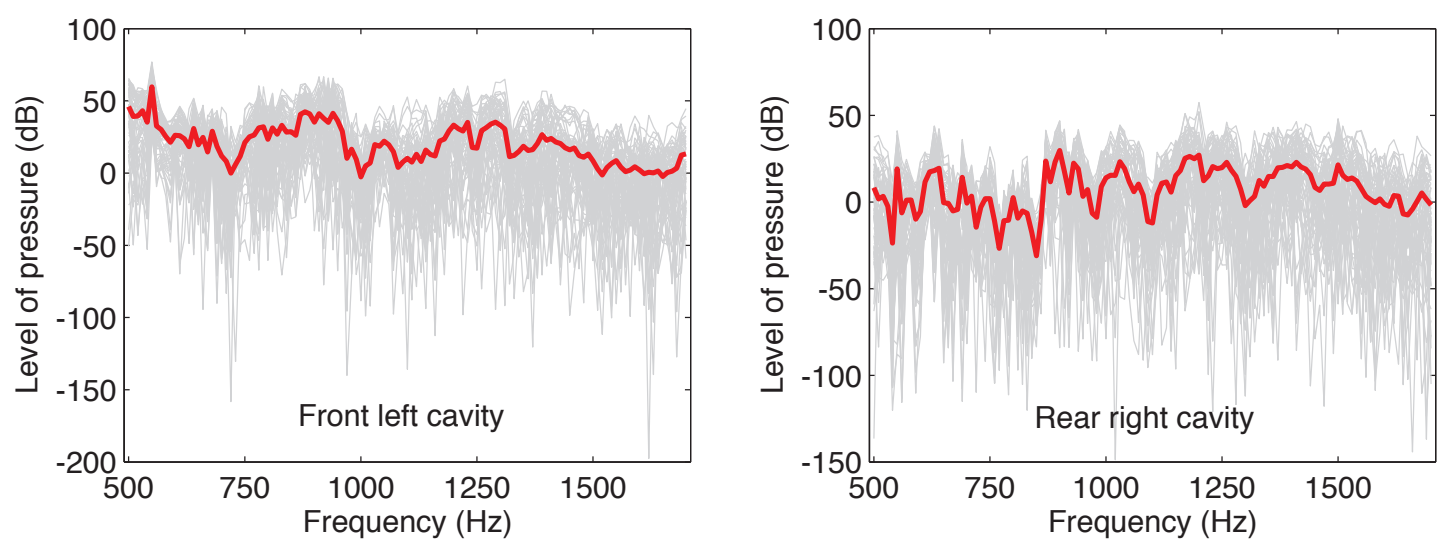

Figure 16. Evolution of the pressure of all the 64 points (grey curves) and the RMS average pressure (red bold line) inside the front left cavity (left) and rear right cavity (right) defined in Figure 12.

studied, from pressure values at 64 evenly-distributed points inside each region, and for the frequency range $[500 \mathrm{~Hz}, 1700 \mathrm{~Hz}]$ sampled every $10 \mathrm{~Hz}$ (the VTCR solution has thus been computed for each sampling frequency). The results are shown in Figure 16, where the RMS average is superposed to the pressure at all 64 evaluation points. Such information may influence the design of the car cavity.

\section{Conclusion}

The extension of the VTCR to 3-D acoustic problems in the medium frequency range was presented. The proposed 3-D acoustic VTCR, based on discretizing the amplitude portrait using spherical harmonics expansions, is the natural generalization of the previouslydeveloped 2-D Fourier VTCR ${ }^{27}$. Various numerical experiments have been performed, demonstrating the efficiency and accuracy of the VTCR and its satisfactory convergence properties. Moreover, an estimation of the energy of the basis functions was shown to permit (like with the previous 2D Fourier version) an effective a priori selection method for the discretization parameter in each sub-cavity, and also to lead to a natural definition of rescaled amplitude portraits that allow to estimate the directivity of the pressure field. Future developments around this approach will include the development of a fully analytical quadrature scheme, the handling of stochastic excitations, the inclusion of approximate radiation conditions at infinity and the extension of the VTCR framework to electromagnetic waves. Future work will also address fundamental issues such as the numerical analysis of the VTCR and the study of its complexity.

\section{Acknowledgements}

The authors gratefully acknowledge the ITN Marie Curie project GA-214909 "MIDFREQUENCY - CAE Methodologies for Mid-Frequency Analysis in Vibration and Acoustics". 


\section{Bibliography}

1. O. C. Zienkiewicz, The Finite Element Method (McGraw-Hill, London, 1977).

2. A. Deraemaeker, I. Babuska and P. Bouillard, Dispersion and pollution of the FEM solution for the Helmholtz equation in one, two and three dimensions, Int. J. Numer. Meth. Eng. 46 (1999) 471-499.

3. C. Soize. Reduced models in the medium frequency range for the general dissipative structural dynamic systems. Eur. J. Mech. A/Solids 17 (1998) 657-685.

4. I. Harari and T.J.R. Hughes. Galerkin/least-squares finite element methods for the reduced wave equation with non- reflecting boundary conditions in unbounded domains, Comput. Meth. Appl. Mech. Eng. 98 (1992) 411-454.

5. I. Babuska, F. Ihlenburg, E.T. Paik and S.A. Sauter. A generalized finite element method for solving the Helmholtz equation in two dimensions with minimal pollution, Comput. Meth. Appl. Mech. Eng., 128 (1995) 325-359.

6. J.M. Melenk and I. Babuska. Approximation with harmonic and generalized harmonic polynomials in the partition of unity method. Comput. Assist. Mech. Eng. Sci. 4 (1997) 607-632.

7. T. Strouboulis, K. Copps and I. Babuska. The generalized finite element method: an example of its implementation and illustration of its performance, Int. J. Numer. Meth. Eng. 47 (2000) 1401-1417.

8. T.J.R. Hughes. Multiscale phenomena: Greens functions and the dirichlet-to-neumann formulation and subgrid scale models and bubbles and the origins of stabilized methods, Comput. Meth. Appl. Mech. Eng. 127 (1995) 387-401.

9. A. F. D. Loula and D. T. Fernandes. A quasi optimal Petrov-Galerkin method for Helmholtz problem, Int. J. Numer. Meth. Eng. 80 (2009) 1595 - 1622.

10. E. Trefftz, Ein gegenstuck zum ritzschen verfahren, in Second International Congress on Applied Mechanics, Zürich, Switzerland, 1926.

11. T. Strouboulis and R. Hidajat, Partition of unity method for Helmholtz equation: q-convergence for plane-wave and wave- band local bases, Applic. Math. 51 (2006) 181-204.

12. O. Cessenat and B. Despres, Application of an ultra weak variational formulation of elliptic PDEs to the two-dimensional Helmholtz problem, SIAM J. Numer. Anal. 35 (1998) 255-299.

13. P. Monk and D.Q. Wang, A least-squares method for the Helmholtz equation, Comput. Meth. Appl. Mech. Eng. 175 (1999) 121-136.

14. C. Farhat, I. Harari and L.P. Franca, The discontinuous enrichment method, Comput. Meth. Appl. Mech. Eng. 190 (2001) 6455-6479.

15. P. Bouillard and S. Suleau, Element-free Galerkin solutions for Helmholtz problems: formulation and numerical assessment of the pollution effect, Comput. Meth. Appl. Mech. Eng. 162 (1998) 317-335, 1998.

16. E. Perrey-Debain, J. Trevelyan and P. Bettess, Wave boundary elements: a theoretical overview presenting applications in scattering of short waves, Eng. Anal. Bound. Elem. 28 (2004) 131-141.

17. W. Desmet, P. Sas and D. Vandepitte, An indirect trefftz method for the steady-state dynamic analysis of coupled vibro-acoustic systems, Comput. Assist. Mech. Eng. Sci. 8 (2001) 271-288.

18. P. Ladevèze, A new computational approach for structure vibrations in the medium frequency range, C.R. Acad. Sci. Paris, série II 322 (1996) 849-856.

19. P. Ladevèze, L. Arnaud, P. Rouch and C. Blanzé, The variational theory of complex rays for the calculation of medium- frequency vibrations, Engineering Computations 18 (2001) 193-214.

20. R. Rouch and P. Ladevèze, The variational theory of complex rays: a predictive tool for mediumfrequency vibrations, Comput. Meth. Appl. Mech. Eng. 192 (2003) 3301-3315.

21. H. Riou, P. Ladevèze and P. Rouch, Extension of the variational theory of complex rays to shells for medium-frequency vibrations, J. Sound. Vibr. 272 (2004) 341-360. 
22. P. Ladevèze, L. Blanc, P. Rouch and C. Blanzé. A multiscale computational method for mediumfrequency vibrations of assemblies of heterogeneous plates, Comput. Struct. 81 (2003) 1267-1276.

23. P. Ladevèze, P. Rouch, H. Riou and X. Bohineust, Analysis of medium-frequency vibrations in a frequency range, J. Comput. Acoust. 11 (2003) 255-284.

24. P. Ladevèze and M. Chevreuil, A new computational method for transient dynamics including the low- and the medium- frequency ranges, Int. J. Numer. Meth. Eng. 64 (2005) 503-527.

25. H. Riou, P. Ladevèze and B. Sourcis, The multiscale VTCR approach applied to acoustics problems, J. Comput. Acoust. 16 (2008) 487-505.

26. H. Riou, P. Ladevèze, B. Sourcis, B. Faverjon and L. Kovalevsky, An adaptive numerical strategy for the medium-frequency analysis of Helmholtz's problem, J. Comput. Acoust. 2 (2012).

27. L. Kovalevsky, P. Ladevèze and H. Riou, The Fourier version of the Variational Theory of Complex Rays for medium-frequency acoustics, Comput. Meth. Appl. Mech. Eng. 225-228 (2012) $142-153$.

28. D. Colton and R. Kress, On the denseness of Herglotz wave functions and electromagnetic Herglotz pairs in Sobolev spaces, Math. Meth. Appl. Sci. 24 (2001) 1289-1303.

29. W. Desmet, B. van Hal, P. Sas and D. Vandepitte, A computationally efficient prediction technique for the steady-state dynamic analysis of coupled vibro-acoustic systems, Advances Eng. Softw. 33 (2002) 527-540.

30. B. Sourcis, Vers une stratégie adaptative de calcul pour la théorie variationnelle des rayons complexes: application à l'acoustique linéaire. PhD thesis, ENS Cachan, France (2009).

31. I. S. Gradshteyn and I. M. Ryzhik. Tables of integrals, series and products ('7th edition). A. Jeffreys and D. Zwillinger, editors (Elsevier, 2007).

32. D. Colton and R. Kress, Inverse acoustic and electromagnetic scattering theory (Springer-Verlag, 1992).

33. F.W.J. Olver, D.W. Lozier, R.F. Boisvert and C.W. Clark (editors). NIST handbook of mathematical functions (Cambridge, 2010). 